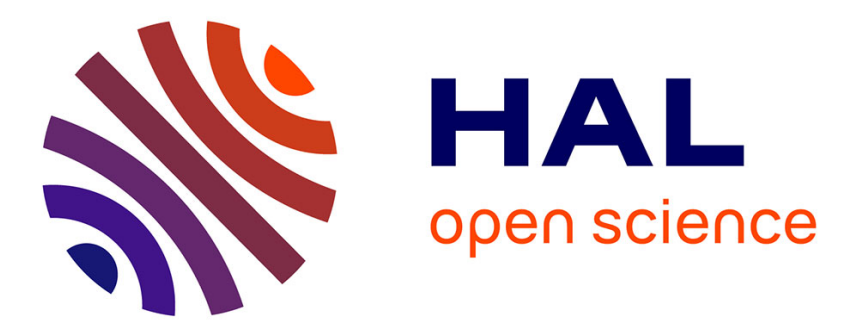

\title{
Novel quasi-scorpionate ligand structures based on a bis-N-heterocyclic carbene chelate core: synthesis, complexation and catalysis
}

\author{
Kingsley J Cavell, Benjamin D Ward, Sedat Yasar, Benson Kariuki
}

\section{To cite this version:}

Kingsley J Cavell, Benjamin D Ward, Sedat Yasar, Benson Kariuki. Novel quasi-scorpionate ligand structures based on a bis-N-heterocyclic carbene chelate core: synthesis, complexation and catalysis. Applied Organometallic Chemistry, 2011, 25 (5), pp.374. 10.1002/aoc.1773 . hal-00627534

\section{HAL Id: hal-00627534 \\ https://hal.science/hal-00627534}

Submitted on 29 Sep 2011

HAL is a multi-disciplinary open access archive for the deposit and dissemination of scientific research documents, whether they are published or not. The documents may come from teaching and research institutions in France or abroad, or from public or private research centers.
L'archive ouverte pluridisciplinaire HAL, est destinée au dépôt et à la diffusion de documents scientifiques de niveau recherche, publiés ou non, émanant des établissements d'enseignement et de recherche français ou étrangers, des laboratoires publics ou privés. 


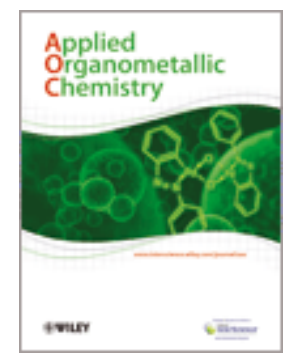

\section{Novel quasi-scorpionate ligand structures based on a bis-N- heterocyclic carbene chelate core: synthesis, complexation and catalysis}

\begin{tabular}{|c|c|}
\hline Journal: & Applied Organometallic Chemistry \\
\hline Manuscript ID: & AOC-10-0190.R1 \\
\hline Wiley - Manuscript type: & Research Article \\
\hline $\begin{array}{r}\text { Date Submitted by the } \\
\text { Author: }\end{array}$ & 25-Nov-2010 \\
\hline Complete List of Authors: & $\begin{array}{l}\text { Cavell, Kingsley; Cardiff University, School of Chemistry } \\
\text { Ward, Benjamin; Cardiff University, Chemistry } \\
\text { Yasar, Sedat; Cardiff University, Chemistry } \\
\text { Kariuki, Benson; Cardiff University, School of Chemistry }\end{array}$ \\
\hline Keywords: & $\begin{array}{l}\text { quasi-scorpionate ligands, N-heterocyclic carbenes, synthesis, } \\
\text { complexes, catalysis }\end{array}$ \\
\hline
\end{tabular}

\section{SCHOLARONE ${ }^{\text {TM }}$ Manuscripts}




\author{
Novel quasi-scorpionate ligand structures based on a bis-N-heterocyclic carbene \\ chelate core: synthesis, complexation and catalysis \\ Sedat Yasar, ${ }^{\text {a,b }}$ Kingsley J. Cavell, ${ }^{* a}$ Benjamin D. Ward, ${ }^{\text {a }}$ Benson Kariuki ${ }^{\text {a }}$ \\ ${ }^{a}$ School of Chemistry, Cardiff University, Main Building, Park Place, Cardiff CF10 3AT, \\ UK; emailCavellKJ@cardiff.ac.uk \\ ${ }^{b}$ Gaziosmanpasa University, Faculty of Science and Arts, Department of Chemistry, \\ 60150, Tokat, TURKEY
}

\begin{abstract}
A series of novel quasi-scorpionate $\mathrm{CNC}$ donor ligands, $\mathrm{MeC}\left(2-\mathrm{C}_{5} \mathrm{H}_{4} \mathrm{~N}\right)\left\{\mathrm{CH}_{2}\right.$ (imidazole$\mathrm{R})(\mathrm{R}=$ methyl, n-butyl, n-propenyl), in which a chelating bis(NHC) core is supplemented by a hemi-labile pyridyl donor, have been prepared. The coordination chemistry of these ligands has been investigated with silver, palladium, rhodium, and iridium. The single crystal $\mathrm{X}$-ray structures of $\left[\mathrm{Rh}\left(\mathrm{NC}_{2}{ }^{\mathrm{Me}}\right)(\mathrm{COD})\right] \mathrm{Cl} \mathbf{8 a}$ and $\left[\operatorname{Ir}\left(\mathrm{NC}_{2}{ }^{\mathrm{Pr}}\right)(\mathrm{COD})\right] \mathrm{Br} \mathbf{9 b}$ have been determined. The catalytic potential of the rhodium and iridium complexes has been assessed in the transfer hydrogenation of ketones; the iridium complexes, which show superior performance, form very effective and stable catalysts.
\end{abstract}

\title{
Introduction
}

The rational design of functional, yet robust molecular complexes relies heavily on the availability of kinetically inert chelating ligands. Metal complexes thus prepared have found widespread application in many diverse areas, from catalysis to biomedical imaging. Consequently, the continued search for new functional complexes must also incorporate the design of novel, readily adaptable supporting ligand environments. Scorpionate ligands are one such group that have an important role in this field. ${ }^{1-3}$ Scorpionate ligands possessing two amide donors, and a third (and possibly a fourth) pyridine or amine donor (Scheme 1), ${ }^{4-8}$ have been shown to support metal complexes which exhibit a range of unusual reactivity, often allowing catalytic intermediates to be 
isolated and studied to an unprecedented level of detail. ${ }^{9}$ Peris and coworkers have prepared an interesting tripodal bis(imidazolylidene) with a phenoxy moiety providing the third donor (Scheme 1). ${ }^{10(a)}$ Such a ligand is extremely strongly bound, and for octahedral metals provides three cis-arranged coordination sites available for further chemistry. In a second paper Peris and coworkers have also reported a bis-carbene ligand containing a pyridine donor on the backbone. ${ }^{10(b)}$ However, the rigid structure of this ligand would probably preclude it from coordinating in a tridentate fashion. Other tridentate NHC based ligands with labile donors, have also been reported, for example see Hahn and Oro et al. ${ }^{10(\mathrm{c}), 11}$

\section{Scheme 1: Selected amido- and NHC-based scorpionate ligands}

N-Heterocyclic carbenes (NHCs) are now well established as powerful co-ordinating ligands that provide a very effective base on which to build new polydentate ligands. Very many publications describing NHC ligands and their application in catalysis have appeared in the past twenty years; a number of excellent reviews have now appeared outlining the synthesis, and chemistry of these ligands and their multidentate derivatives. $^{11}$ The field is extremely vibrant and novel developments in NHC ligand design appear regularly. ${ }^{12,13}$

As part of our ongoing investigation into novel functionalised N-heterocyclic carbene ligands as supporting environments for metal complexes and their application in catalysis, we herein report the synthesis and coordination chemistry of chelating bis(NHC) ligands, possessing a pyridyl-donor, with silver, palladium, rhodium, and iridium. Catalytic transfer hydrogenation reactions employing the rhodium and iridium complexes are reported. The pyridyl ring is seen as an interesting structural feature of this ligand, in square-planar complexes the hemi-labile pyridyl donor is well placed to coordinate to the metal centre to stabilise a catalyst resting state, whilst easily decoordinating to allow a reaction to proceed when a lower coordination number is required. 


\section{Results and Discussion}

\section{Ligand synthesis}

The ligands $\mathrm{MeC}\left(2-\mathrm{C}_{5} \mathrm{H}_{4} \mathrm{~N}\right)\left\{\mathrm{CH}_{2}\right.$ (imidazole- $\left.\mathrm{R}\right)(\mathrm{R}=$ methyl, n-butyl, n-propenyl) were prepared according to the procedure depicted in Scheme 2. The di-hydroxy species, 2, was synthesised by the hydroxymethylation of 2-ethylpyridine with aqueous formaldehyde, following the method of $\mathrm{Gade}^{4}$ this gave a mixture of the monohydroxymethylation product $\mathbf{1}$ and the dihydroxymethylation product $\mathbf{2}$. The yield of 2 was increased by reacting 1 with further equivalents of formaldehyde under identical conditions. Treatment of a solution of $\mathbf{2}$ with methanesulfonyl chloride gave the product, $\mathbf{3}$, as a red oil in nearly quantitative yield (93\%); further reaction of $\mathbf{3}$ with dry lithium bromide yielded 4 as pale yellow oil (56\% yield). The bis-imidazolium salts were synthesised by reaction of 4 with selected N-substituted imidazoles to give the imidazolium salts $\left[\mathrm{H}_{2} \mathrm{NC}_{2}{ }^{\mathrm{R}}\right] \mathrm{Br}_{2}(\mathrm{R}=\mathrm{Me}, \mathrm{Bu}, \mathrm{Pr})$ 5a-c, shown in Scheme $2(75-80 \%$ yields).

Scheme 2: Synthesis of the bis-imidazolium ligand precursors

\section{Synthesis of metal complexes}

Silver complexes of the new ligands were prepared by dissolving the bis-imidazolium salts in $\mathrm{CH}_{2} \mathrm{Cl}_{2}$ (DCM) to which $\mathrm{Ag}_{2} \mathrm{O}$ was added (Scheme 3). The resulting suspension was stirred at room temperature overnight, yielding a grey precipitate and red solution. After filtration and washing, the silver complexes $6 \mathbf{a}-\mathbf{c}$ were obtained as white powders (in $65-80 \%$ yield), which rapidly discoloured on standing (the structure depicted in Scheme 3 is based on the molecular formulae obtained from high resolution MS data).

Scheme 3: Synthesis of the Ag-bis-NHC complexes 6a-c 
A common function of Ag-NHC complexes is to act as a ligand transfer agent. To demonstrate the feasibility of such an approach for the current ligands a square-planar bis-NHC-Pd complex was prepared by treating the Pd precursor $\left[\mathrm{PdCl}_{2}\left(\mathrm{NCCH}_{3}\right)_{2}\right]$ with a solution of the Ag complex as shown in (Eq 2, Scheme 4); complex 7 was obtained in good yield as a stable yellow compound. $\mathrm{Rh}$ and Ir complexes were readily prepared from the bis-imidazolium salt, $\mathrm{KN}\left(\mathrm{SiMe}_{3}\right)_{2}$ and the appropriate metal precursor complex, according to $\mathrm{Eq} 3$, Scheme 4.

\section{Scheme 4: Synthesis of transition metal complexes of the bis-imidazole ligands}

\section{Structural characterisation}

The structure of complexes $\left[\mathrm{Rh}\left(\mathrm{NC}_{2}{ }^{\mathrm{Me}}\right)(\mathrm{COD})\right] \mathrm{Cl} \mathbf{8 a}$ and $\left[\mathrm{Ir}\left(\mathrm{NC}_{2}{ }^{\mathrm{Pr}}\right)(\mathrm{COD})\right] \mathrm{Br} \mathbf{9 b}$ were confirmed by crystallographic analyses. The molecular structures of the two complexes are depicted in Figures 1 and 2 respectively, with selected bond lengths and angles provided in Table 1. The precision in the bond lengths and angles is somewhat low. Nevertheless, the two structures confirm the overall connectivity of the ligands, and indicate the coordination mode via the two NHC moieties. In these cases it can be seen that the pyridyl arm remains pendant, presumably owing to the preference for a square planar coordination geometry in these $\mathrm{d}^{8}$ complexes. However, it can be clearly seen that the pyridyl donor lies in a position which makes coordination likely, should the metal require further coordinative saturation, for example, in octahedral complexes, or should coordination be required to stabilise a catalyst resting state. The principal bond lengths and angles (i.e. those associated with the central coordination sphere) are comparable to those reported in the Cambridge Structural Database: $\mathrm{Rh}-\mathrm{C}_{\mathrm{NHC}}=1.901-2.224$, mean 2.038 for 161 examples); $\mathrm{Rh}-\mathrm{C}_{\mathrm{COD}}=1.998-2.397$, mean 2.160 for 1098 examples; Ir$\mathrm{C}_{\mathrm{NHC}}=1.907-2.115$, mean 2.040 for 111 examples; $\mathrm{Ir}-\mathrm{C}_{\mathrm{COD}}=1.984-2.363$, mean 2.165 for 509 examples. ${ }^{14}$ Complex 9b crystallises with two independent molecules in the asymmetric unit; the two show no significant differences, the corresponding metric parameters are provided in parentheses in Table 1. Complex 8a crystallises with a chloride anion, whereas $\mathbf{9 b}$ crystallises with a bromide. Since both complexes were 
prepared using an analogous route, and both halide ions were present (chloride being derived from the metal precursor and bromide being derived from the imidazolium salt), we ascribe this phenomenon to subtle differences in crystal packing. However, neither structure indicates any evidence of interaction between the anion and the cationic complex, and we therefore deem this difference to be of little consequence in relation to the coordination motif of the ligand or the catalytic ability of the complexes.

Figure 1: Molecular structure of $\left.\left[\mathrm{Rh}_{(} \mathrm{NC}_{2}^{\mathrm{Me}}\right)(\mathrm{COD})\right] \mathrm{Cl}(\mathbf{8 a}) . \mathrm{H}$ atoms and anion omitted for clarity, thermal ellipsoids are drawn at $25 \%$ probability

Figure 2: Molecular structure of $\left[\operatorname{Ir}\left(\mathrm{NC}_{2}{ }^{\mathrm{Bu}}\right)(\mathrm{COD})\right] \mathrm{Br}(\mathbf{9 b})$. H atoms, the second crystallographically independent molecule, and anion omitted for clarity, thermal ellipsoids are drawn at $25 \%$ probability

\section{Table 1 here}

\section{Catalytic studies}

To explore the effectiveness of these new ligands as ligands in catalysis, preliminary catalytic investigations were undertaken into the transfer hydrogenation of ketones. Initial results were very promising and good catalytic activity, and catalyst stability was observed.

The performances of Iridium(I) and Rhodium(I) complexes, 8 and $\mathbf{9}$, $\left[\mathrm{M}\left(\mathrm{NC}_{2}{ }^{\mathrm{R}}\right)(\mathrm{COD})\right]^{+} \mathrm{X}^{-}$, were tested using a variety of substrates. The complexes catalyse the hydrogen transfer from ${ }^{i} \mathrm{PrOH}$ with potassium tert-butoxide as the base, according to Eq. 4. As previously observed, ${ }^{15}$ the $\operatorname{Ir}(\mathrm{I})-\mathrm{NHC}$ complexes show higher catalytic activity than their rhodium analogues. With 1 mol\% catalyst loading, each of the Ir complexes gave $100 \%$ conversion in $60 \mathrm{~min}$ or less for all substrates tested, hence lower concentrations of catalyst $(0.3$ and $0.01 \mathrm{~mol} \%)$ were also investigated, and results for the lower loadings are reported in Table 2. $\left[\mathrm{Rh}\left(\mathrm{NC}_{2}{ }^{\mathrm{Me}}\right)(\mathrm{COD})\right] \mathrm{Cl} \mathrm{8a},(0.3 \mathrm{~mol} \%$; the only $\mathrm{Rh}$ 
complex tested) required over $16 \mathrm{hr}$ to yield $100 \%$ conversion of 4-bromoacetophenone into the corresponding alcohol (entry 19, Table 2). Although all iridium complexes gave rise to very effective catalysts, complexes with ligands bearing the longer chain, propenyl and n-butyl groups show better performance than the methyl bearing catalyst system.

\section{Equation 4 here}

Table 2. here

Plots of conversion versus time for $\operatorname{Ir}(\mathrm{I})-\mathrm{NHC}$ complex 9c, with different substrates were investigated using a concentration of $0.3 \mathrm{~mol} \%$ catalyst and the results are shown in Figs 4 and 5. Reactions were monitored by taking aliquots from the reaction mixture at set intervals and the $\%$ conversion determined. Complete conversion of 4bromoacetophenone to the alcohol is achieved after approximately $30 \mathrm{~min}$ (Fig 4).

Figure 3: Plot of conversion versus time for p-bromoacetophenone (1 mmol) catalysed by 9 c $(0.3$ mol\%)

Figure 4: Plot of conversion versus time for p-methylacetophenone (1 mmol) catalysed by 9 c $(0.3$ mol\%)

To test the stability of the catalyst system, catalyst 9c was tested under normal operating conditions, however, after $30 \mathrm{~min}$ (and $\sim 100 \%$ conversion) an additional $1 \mathrm{mmol}$ of $p$ bromoacetophenone was added and the reaction monitored, and then after $100 \mathrm{~min}$ a third aliquot of substrate was added (Results are shown Fig 5). A similar procedure was also followed using $p$-methylacetophenone as substrate. Importantly, results indicate that there is little diminution in catalyst performance with time, despite the reduced catalyst concentration and increasing product concentration.

Figure 5: Lifetime studies for catalyst 9 c (0.3 mol\%) with p-bromocetophenone as substrate 
The catalytic performance of the chelating NHC-Ir complexes, although not optimised in terms of ligand substituents or reaction conditions, generally compare quite favourably with previously reported systems for transfer hydrogenation. ${ }^{15-20}$ The catalyst systems showed good stability and activity for the range of substrate tested.

\section{Conclusions}

A new series of bis(carbene)pyridine ligands have been shown to provide valuable supporting environments for a range of late transition metals. The complexes have been applied as catalysts in transfer hydrogenation, they exhibit good activity and excellent catalyst stability. It is clear that there is potential for these ligands to find applications with many other metals and catalytic processes other than those described here; the CNC coordination motif is expected to facilitate the development of novel organometallic complexes for both fundamental studies as well as in catalytic applications. Further studies are currently underway, to determine the efficacy of the pyridyl coordination with a variety of transition metals, and also in the development of new donor-functionalised ligand structures based on a related bis-NHC core.

\section{Experimental}

All manipulations were performed using standard Schlenk techniques under an argon atmosphere, except where otherwise noted. Complexes $[\mathrm{Rh}(\mathrm{COD}) \mathrm{Cl}]_{2},[\operatorname{Ir}(\mathrm{COD}) \mathrm{Cl}]_{2}$ were synthesised according to literature methods. ${ }^{21} \mathrm{NHC}$ salts and the $\mathrm{Rh}$ and $\mathrm{Ir}$ complexes were prepared as previously reported. ${ }^{13(1)}$ Solvents of analytical grade were freshly distilled from sodium/benzophenone (thf, hexane) or from calcium hydride $\left(\mathrm{CH}_{2} \mathrm{Cl}_{2}\right)$ or dried using a Braun SPS-800 system (hexane, $\mathrm{CH}_{2} \mathrm{Cl}_{2}$ ) or a Vacuum Atmospheres recirculating SPS system (thf). Deuterated solvents for NMR measurements were distilled from the appropriate drying agents under $\mathrm{N}_{2}$ immediately prior to use, following standard literature methods. Air-sensitive compounds were stored and weighed in a glovebox. All reagents (1,3-dibromopropane, 1,4-diiodobutane, 2aminopyridine, o-anisidine, 2,6-dimethylaniline, 2,6-diisopropylaniline, triethylorthoformate, sodium tetrafluoroborate, and potassium bis(trimethylsilyl)amide) wereobtained from commercial suppliers and used as received. ${ }^{1} \mathrm{H}$ and ${ }^{13} \mathrm{C}$ NMR spectra were obtained on Bruker Advance AMX 400, 500 or Jeol Eclipse 300 spectrometers. 
Chemical shifts $(\delta)$ were expressed in ppm downfield from tetramethylsilane using the residual protio solvent $\left({ }^{1} \mathrm{H}\right)$ or solvent $\left({ }^{13} \mathrm{C}\right)$ as an internal standard $\left(\mathrm{CDCl}_{3},{ }^{1} \mathrm{H} 7.26 \mathrm{ppm}\right.$ and ${ }^{13} \mathrm{C} 77.0$ ppm; benzene- $\mathrm{d}_{6}{ }^{1} \mathrm{H} 7.15 \mathrm{ppm}$ and $\left.{ }^{13} \mathrm{C} 128.0 \mathrm{ppm}\right)$. Coupling constants $\mathrm{J}$ are expressed in Hertz. HRMS were obtained on a Waters LCT Premier XE instrument and are reported as $\mathrm{m} / \mathrm{z}$ (relative intensity). Infrared spectra were recorded using a JASCO FT/IR-660 Plus spectrometer and analysed in solution (dichloromethane).

\section{Synthesis of 2-(1,3-dihydroxy-2-methylpropan-2-yl)pyridine, 2}

Compound 2 was synthesised following the method of Gade et $a l^{4}$ using a mixture of 2ethylpyridine $(37.5 \mathrm{ml}, 35.2 \mathrm{~g}, 0.327 \mathrm{~mol})$ and an aqueous solution of formaldehyde $(37 \%, 115.5 \mathrm{~mL}, 1.60 \mathrm{~mol})$. As described by Gade, the total yield of 2 was increased to $48 \%$ by reaction of 1 with a further 4 equivalents of formaldehyde under the same reaction conditions. ${ }^{1} \mathrm{H} \mathrm{NMR}\left(400.1 \mathrm{MHz}, \mathrm{CDCl}_{3}, 293 \mathrm{~K}\right) \delta 1.16\left(\mathrm{~s}, \mathrm{CCH}_{3}, 3 \mathrm{H}\right), 3.74(\mathrm{~d}$, $\left.J=11.1 \mathrm{~Hz}, \mathrm{CCH}_{3} \mathrm{CH}_{2}, 2 \mathrm{H}\right), 3.94\left(\mathrm{~d}, J=11.1 \mathrm{~Hz}, \mathrm{CHCH}_{3} \mathrm{CH}_{2}, 2 \mathrm{H}\right), 7.14(\mathrm{~d}, J=7.6 \mathrm{~Hz}$, $\left.\mathrm{C}_{5} \mathrm{H}_{4} \mathrm{~N}, 1 \mathrm{H}\right), 7.27\left(\mathrm{~m}, \mathrm{C}_{5} \mathrm{H}_{4} \mathrm{~N}, 1 \mathrm{H}\right), 7.66\left(\mathrm{~m}, \mathrm{C}_{5} \mathrm{H}_{4} \mathrm{~N}, 1 \mathrm{H}\right), 8.43\left(\mathrm{~d}, \mathrm{C}_{5} \mathrm{H}_{4} \mathrm{~N}, 1 \mathrm{H}\right) \mathrm{ppm}$. ${ }^{13} \mathrm{C}\left\{{ }^{1} \mathrm{H}\right\} \mathrm{NMR}\left(100.6 \mathrm{MHz}, \mathrm{CDCl}_{3}, 293 \mathrm{~K}\right) \delta 19.7,45.7,69.3,121.5,121.7,137.0,147.8$, $165.1 \mathrm{ppm}$.

\section{Synthesis of 2-(1,3-dibromo-2-methylpropan-2-yl)pyridine, 4}

This compound was synthesised in a two-step process.

Part (a): Synthesis of 2-[bis-1,3-methylsulfonate-2-methylpropan-2-yl)]pyridine, 3

A solution of $2(16.7 \mathrm{~g}, 0.1 \mathrm{~mol})$ and triethylamine $(62.6 \mathrm{~mL}, 0.45 \mathrm{~mol})$ in dichloromethane $(200 \mathrm{~mL})$ was cooled to $-2{ }^{\circ} \mathrm{C}$, and a solution of methanesulfonyl chloride $(19.3 \mathrm{~mL}, 0.25 \mathrm{~mol})$ in dichloromethane $(50 \mathrm{~mL})$ added dropwise, taking care that the temperature did not exceed $+2{ }^{\circ} \mathrm{C}$. The resulting suspension was warmed to room temperature and stirred for one hour. The reaction product was washed successively with hydrochloric acid $(1 \mathrm{M}, 1 \times 50 \mathrm{~mL})$, water $(1 \times 50 \mathrm{~mL})$, sodium carbonate solution $(1 \times 50$ $\mathrm{mL})$, brine $(1 \times 50 \mathrm{~mL})$ and finally again with water $(1 \times 50 \mathrm{~mL})$. After drying over $\mathrm{Na}_{2} \mathrm{SO}_{4}$ and evaporating the solvent, the residue was dried in vacuo to yield $\mathbf{3}$ as a red oil which was used without further purification $(29.3 \mathrm{~g}, 93 \%) .{ }^{1} \mathrm{H} \mathrm{NMR}\left(400.1 \mathrm{MHz}, \mathrm{CDCl}_{3}, 293\right.$ $\mathrm{K}): \delta 1.50\left(\mathrm{~s}, \mathrm{CCH}_{3}, 3 \mathrm{H}\right), 2.96\left(\mathrm{~s}, \mathrm{CH}_{2} \mathrm{SO}_{2} \mathrm{CH}_{3}, 6 \mathrm{H}\right), 4.63-4.52(\mathrm{dd}, \mathrm{AB}, J=8.8 \mathrm{~Hz}$, 
$\left.\mathrm{CCH}_{3} \mathrm{CH}_{2}, 4 \mathrm{H}\right), 7.21\left(\mathrm{~d}, J=7.9 \mathrm{~Hz}, \mathrm{C}_{5} \mathrm{H}_{4} \mathrm{~N}, 1 \mathrm{H}\right), 7.27\left(\mathrm{~d}, J=7.9 \mathrm{~Hz}, \mathrm{C}_{5} \mathrm{H}_{4} \mathrm{~N}, 1 \mathrm{H}\right), 7.70$ $\left(\mathrm{t}, J=8.0 \mathrm{~Hz}, \mathrm{C}_{5} \mathrm{H}_{4} \mathrm{~N}, 1 \mathrm{H}\right), 8.49\left(\mathrm{~d}, J=8.0 \mathrm{~Hz}, \mathrm{C}_{5} \mathrm{H}_{4} \mathrm{~N}, 1 \mathrm{H}\right) \mathrm{ppm} .{ }^{13} \mathrm{C}\{1 \mathrm{H}\} \mathrm{NMR}(100.6$ $\left.\mathrm{MHz}, \mathrm{CDCl}_{3}, 293 \mathrm{~K}\right) \delta 19.1,36.7,36.8,45.1,72.7,119.1,137.5,158.4,163.3$ ppm.

Part (b): Synthesis of 4

Compound 3 (31.6 g, $0.1 \mathrm{~mol}$ ) was dissolved in anhydrous dimethyl sulfoxide (150 mL) and heated to $70{ }^{\circ} \mathrm{C}$. Vigorously dried (vacuo, $100{ }^{\circ} \mathrm{C}, 3 \mathrm{~d}$ ) lithium bromide $(21.75 \mathrm{~g}$, $0.25 \mathrm{~mol}$ ) was then added in one portion, and the solution stirred for two days at $70{ }^{\circ} \mathrm{C}$. After cooling to room temperature, water $(200 \mathrm{~mL})$ was added, and the mixture stirred for $30 \mathrm{~min}$. The milky solution was extracted with diethyl ether $(7 \times 70 \mathrm{~mL})$, and the combined ether extracts washed with water $(3 \times 50 \mathrm{~mL})$ to remove residual dimethyl sulfoxide. The organic phase was separated, dried with $\mathrm{Na}_{2} \mathrm{SO}_{4}$, and the solvent evaporated to give an oily, crude product. Column chromatography $\left(\mathrm{SiO}_{2}, 0.060-0.200\right.$ $\mathrm{mm}$, pore diameter ca. $6 \mathrm{~nm})$ using a combination of ethyl acetate and hexane $(3: 1)$ as eluent yielded compound 4 as a light yellow oil (16.4 g, $56 \%)$. ${ }^{1} \mathrm{H}$ NMR (400.1 MHz, $\left.\mathrm{CDCl}_{3}, 293 \mathrm{~K}\right): \delta 1.63\left(\mathrm{~s}, \mathrm{CCH}_{3}, 3 \mathrm{H}\right), 4.00-3.86\left(\mathrm{dd}, \mathrm{AB}, J=10.0 \mathrm{~Hz}, \mathrm{CCH}_{3} \mathrm{CH}_{2}, 4 \mathrm{H}\right)$, $7.18\left(\mathrm{~d}, J=7.9 \mathrm{~Hz}, \mathrm{C}_{5} \mathrm{H}_{4} \mathrm{~N}, 1 \mathrm{H}\right), 7.23\left(\mathrm{~d}, J=7.9 \mathrm{~Hz}, \mathrm{C}_{5} \mathrm{H}_{4} \mathrm{~N}, 1 \mathrm{H}\right), 7.66(\mathrm{t}, J=7.9 \mathrm{~Hz}$, $\left.\mathrm{C}_{5} \mathrm{H}_{4} \mathrm{~N}, 1 \mathrm{H}\right), 8.54\left(\mathrm{~d}, J=8.0 \mathrm{~Hz}, \mathrm{C}_{5} \mathrm{H}_{4} \mathrm{~N}, 1 \mathrm{H}\right) \mathrm{ppm} .{ }^{13} \mathrm{C}\left\{{ }^{1} \mathrm{H}\right\}$ NMR (100.6 MHz, $\mathrm{CDCl}_{3}$, $293 \mathrm{~K}): \delta 23.1,42.1,46.0,118.3,118.4,137.1,160.0,164.1 \mathrm{ppm}$. HR-MS (ES), $\mathrm{m} / z=$ $291.9345[\mathrm{M}+\mathrm{H}]^{+}$(calc. for $\mathrm{C}_{9} \mathrm{H}_{12} \mathrm{Br}_{2} \mathrm{~N}$ : 291.9336).

\section{Synthesis of 2-(2-methyl-1,3-bis(3-methylimidazolidin-1-yl)propan-2-yl)pyridine bromide, 5 a}

2-(1,3-dibromo-2-methylpropan-2-yl)pyridine (2.93 g, $10 \mathrm{mmol})$ and 1-methylimidazole (1.97 g, $24 \mathrm{mmol})$ were stirred at $100{ }^{\circ} \mathrm{C}$ overnight. The resulting hygroscopic brown solid was washed with diethyl ether and dried under vacuum to give the pure salt 5a (3.65 g, 80\%). ${ }^{1} \mathrm{H}$ NMR (400.1 MHz, D $\left.2 \mathrm{O}, 293 \mathrm{~K}\right): \delta 1.43\left(\mathrm{~s}, \mathrm{CCH}_{3}, 3 \mathrm{H}\right), 3.82\left(\mathrm{~s}, \mathrm{NCH}_{3}, 6 \mathrm{H}\right)$, 4.61-4.86 (dd, $\mathrm{AB}, J=11.2 \mathrm{~Hz}, \mathrm{CCH}_{3} \mathrm{CH}_{2}, 4 \mathrm{H}$ ), 7.25 (s, $\mathrm{NCHCHN}, 2 \mathrm{H}$ ), 7.65 (s, $\mathrm{NCHCHN}, 2 \mathrm{H}), 7.21\left(\mathrm{~m}, \mathrm{C}_{5} \mathrm{H}_{4} \mathrm{~N}, 1 \mathrm{H}\right), 7.38\left(\mathrm{~m}, \mathrm{C}_{5} \mathrm{H}_{4} \mathrm{~N}, 1 \mathrm{H}\right), 7.85\left(\mathrm{~m}, \mathrm{C}_{5} \mathrm{H}_{4} \mathrm{~N}, 1 \mathrm{H}\right), 8.7$ (t, $\left.J=3.2 \mathrm{~Hz}, \mathrm{C}_{5} \mathrm{H}_{4} \mathrm{~N}, 1 \mathrm{H}\right), 9.06(\mathrm{~s}, \mathrm{NCHN}, 2 \mathrm{H}) \mathrm{ppm} .{ }^{13} \mathrm{C}\left\{{ }^{1} \mathrm{H}\right\} \mathrm{NMR}\left(100.6 \mathrm{MHz}, \mathrm{D}_{2} \mathrm{O}, 293\right.$ $\mathrm{K}): 17.8,35.8,46.6,56.2,122.7,123.4,124.3,138.6,139.0,148.9,149.8,156.9$ ppm. HR-MS (ES), $m / z=376.1127[\mathrm{M}+\mathrm{Br}]^{+}$(calc. for $\mathrm{C}_{17} \mathrm{H}_{23} \mathrm{BrN}_{5}: 376.1137$ ). 


\section{Synthesis of 2-(1,3-bis(3-butylimidazolidin-1-yl)-2-methylpropan-2-yl)pyridine bromide, $5 b$}

This compound was prepared an analogous manner to 5a; using 1-butylimidazole (2.98 g, $24 \mathrm{mmol})$. The resulting hygroscopic white solid was washed with diethyl ether and dried under vacuum to give the salt $5 \mathbf{b}$ (4 g, 75\%). ${ }^{1} \mathrm{H}$ NMR (400.1 MHz, DMSO-d 6,293 $\mathrm{K}): \delta 0.84\left(\mathrm{t}, J=7.6 \mathrm{~Hz}, \mathrm{NCH}_{2} \mathrm{CH}_{2} \mathrm{CH}_{2} \mathrm{CH}_{3}, 6 \mathrm{H}\right), 1.09\left(\mathrm{~m}, \mathrm{NCH}_{2} \mathrm{CH}_{2} \mathrm{CH}_{2} \mathrm{CH}_{3}, 4 \mathrm{H}\right), 1.42$ (s, $\left.\mathrm{CCH}_{3}, 3 \mathrm{H}\right), 1.66\left(\mathrm{~m}, \mathrm{NCH}_{2} \mathrm{CH}_{2} \mathrm{CH}_{2} \mathrm{CH}_{3}, 4 \mathrm{H}\right), 4.11\left(\mathrm{t}, \mathrm{J}=7.2 \mathrm{~Hz}, \mathrm{NCH}_{2} \mathrm{CH}_{2} \mathrm{CH}_{2} \mathrm{CH}_{3}\right.$, 4H), 4.65-4.85 (dd, AB, J=13.6 Hz, $\mathrm{CCH}_{3} \mathrm{CH}_{2}, 4 \mathrm{H}$ ), 7.37 (s, NCHCHN, 2H), 7.4 (m, $\left.\mathrm{C}_{5} \mathrm{H}_{4} \mathrm{~N}, 1 \mathrm{H}\right), 7.6\left(\mathrm{~s}, \mathrm{C}_{5} \mathrm{H}_{4} \mathrm{~N}, 1 \mathrm{H}\right), 7.74(\mathrm{~s}, \mathrm{NCHCHN}, 2 \mathrm{H}), 7.85\left(\mathrm{~m}, \mathrm{C}_{5} \mathrm{H}_{4} \mathrm{~N}, 1 \mathrm{H}\right), 8.7$ (d, $\left.J=1.2 \mathrm{~Hz}, \mathrm{C}_{5} \mathrm{H}_{4} \mathrm{~N}, 1 \mathrm{H}\right), 8.97(\mathrm{~s}, \mathrm{NCHN}, 2 \mathrm{H}) \mathrm{ppm} .{ }^{13} \mathrm{C}\left\{{ }^{1} \mathrm{H}\right\} \mathrm{NMR}\left(100.6 \mathrm{MHz}, \mathrm{DMSO}-\mathrm{d}_{6}\right.$, $293 \mathrm{~K}): \delta 13.8,19.4,23.9,31.1,38.9,51.48,55.8,113.7,121.4,123.0,123.4,136.0$, $148.1,159.7,160.2 \mathrm{ppm}$.

\section{Synthesis of 2-(1,3-bis(3-propenylimidazolidin-1-yl)-2-methylpropan-2-yl)pyridine bromide, 5c}

This compound was prepared an analogous manner to 5a; from 1-propenylimidazol (2.6 g, $24 \mathrm{mmol}$ ). The resulting hygroscopic brown solid was washed with diethyl ether and dried under vacuum to give the salt 5 c (3.97 g, 78 \%). ${ }^{1} \mathrm{H}$ NMR (400.1 MHz, DMSO-d $\mathrm{d}_{6}$, $293 \mathrm{~K}): \delta 1.43\left(\mathrm{~s}, \mathrm{CCH}_{3}, 3 \mathrm{H}\right), 4.82$ (d, $\left.J=6.8 \mathrm{~Hz}, \mathrm{NCH}_{2} \mathrm{CHCH}_{2}, 4 \mathrm{H}\right), 4.63-4.89$ (dd, AB, $\left.J=13.6 \mathrm{~Hz}, \mathrm{CCH}_{3} \mathrm{CH}_{2}, 4 \mathrm{H}\right), 5.32$ (d, $\left.J=0.8 \mathrm{~Hz}, \mathrm{NCH}_{2} \mathrm{CHCH}_{2}, 4 \mathrm{H}\right), 5.97$ (m, $\left.\mathrm{NCH}_{2} \mathrm{CHCH}_{2}, 2 \mathrm{H}\right), 6.9$ (s, NCHCHN, 2H), 7.34 (s, NCHCHN, 2H), $7.38\left(\mathrm{~m}, \mathrm{C}_{5} \mathrm{H}_{4} \mathrm{~N}\right.$, $1 \mathrm{H}), 7.68\left(\mathrm{~s}, \mathrm{C}_{5} \mathrm{H}_{4} \mathrm{~N}, 1 \mathrm{H}\right), 7.82\left(\mathrm{~m}, \mathrm{C}_{5} \mathrm{H}_{4} \mathrm{~N}, 1 \mathrm{H}\right), 8.7$ (t, J=1.2 Hz, $\left.\mathrm{C}_{5} \mathrm{H}_{4} \mathrm{~N}, 1 \mathrm{H}\right), 9.03$ (s, $\mathrm{NCHN}, 2 \mathrm{H}) \mathrm{ppm} .{ }^{13} \mathrm{C}\left\{{ }^{1} \mathrm{H}\right\} \mathrm{NMR}\left(100.6 \mathrm{MHz}, \mathrm{DMSO}_{6}, 293 \mathrm{~K}\right): \delta 20.6,39.5,46.6$, $51.3,56.0,120.3,122.8,123.7,124.2,132.2,137.6,149.3,149.8,158.5$ ppm.

\section{Synthesis of $\mathrm{Ag}(\mathrm{I})-\mathrm{NHC}$ Complexes}

Synthesis of 2-(2-methyl-1,3-bis(3-methylimidazolidin-1-yl)propan-2-yl)pyridine Silver(I) bromide, 6 a

2-(2-methyl-1,3-bis(3-methylimidazolidin-1-yl)propan-2-yl)pyridine bromide, 5a, (0.45 g, $1 \mathrm{mmol})$ was dissolved in dried DCM $(20 \mathrm{~mL})$ and $\operatorname{Ag}_{2} \mathrm{O}(0.271,1 \mathrm{mmol})$ added. The 
resulting suspension was stirred at room temperature for overnight, yielding a gray precipitate and red solution. The supernatant was separated and stripped in vacuo and the red solid washed with ether and hexane. The resultant white powder was dried in vacuo but rapidly discoloured, giving $6 \mathbf{a}(0.37 \mathrm{~g}, 75 \%)$ a red creamy powder that showed no further signs of decomposition at room temperature. ${ }^{1} \mathrm{H}$ NMR $\left(400.1 \mathrm{MHz}, \mathrm{D}_{2} \mathrm{O}, 293 \mathrm{~K}\right)$ : $\delta 1.55$ (s, $\left.\mathrm{CCH}_{3}, 3 \mathrm{H}\right), 3.76\left(\mathrm{~s}, \mathrm{NCH}_{3}, 6 \mathrm{H}\right), 4.73-4.93$ (dd, $\mathrm{AB}, J=13.6 \mathrm{~Hz}, \mathrm{CCH}_{3} \mathrm{CH}_{2}$, 4H), 6.8 (s, NCHCHN, 2H), $7.3\left(\mathrm{~d}, J=3.2 \mathrm{~Hz}, \mathrm{C}_{5} \mathrm{H}_{4} \mathrm{~N}, 1 \mathrm{H}\right), 7.42$ (s, NCHCHN, 2H), $7.43\left(\mathrm{~m}, \mathrm{C}_{5} \mathrm{H}_{4} \mathrm{~N}, 1 \mathrm{H}\right), 7.80\left(\mathrm{t}, J=2.4 \mathrm{~Hz}, \mathrm{C}_{5} \mathrm{H}_{4} \mathrm{~N}, 1 \mathrm{H}\right), 8.75\left(\mathrm{~d}, J=1.6 \mathrm{~Hz}, \mathrm{C}_{5} \mathrm{H}_{4} \mathrm{~N}, 1 \mathrm{H}\right)$ ppm. ${ }^{13} \mathrm{C}\left\{{ }^{1} \mathrm{H}\right\}$ NMR (100.6 MHz, D $\left.2 \mathrm{O}, 293 \mathrm{~K}\right), 19.7,34.8,46.7,54.9,58.4,64.9,122.5$, $122.6,122.8,137.5,149.5,160.0,181.7$ ppm. ES-MS: $m / z=484(20 \%)[\mathrm{M}+\mathrm{H}]^{+}, 402$ $(100 \%)[\mathrm{M}-\mathrm{Br}]^{+}$.

\section{Synthesis of 2-(1,3-bis(3-butylimidazolidin-1-yl)-2-methylpropan-2-yl)pyridine Silver(I) bromide, 6 b.}

This compound was prepared an analogous manner to $\mathbf{6 a}$; from 2-(1,3-bis(3butylimidazolidin-1-yl)-2-methylpropan-2-yl)pyridine bromide, $5 \mathbf{b}$ (0.54 g, $1 \mathrm{mmol})$ and $\mathrm{Ag}_{2} \mathrm{O}(0.27 \mathrm{~g}, 1 \mathrm{mmol})$. The resultant solid was dried in vacuo giving $6 \mathbf{b}(0.37 \mathrm{~g}, 66 \%)$ as a grey creamy powder. ${ }^{1} \mathrm{H}$ NMR $\left(400.1 \mathrm{MHz}, \mathrm{DMSO}_{6}, 293 \mathrm{~K}\right): \delta 0.74(\mathrm{t}, J=6 \mathrm{~Hz}$, $\left.\mathrm{NCH}_{2} \mathrm{CH}_{2} \mathrm{CH}_{2} \mathrm{CH}_{3}, 6 \mathrm{H}\right), 1.09$ (m, $\left.\mathrm{NCH}_{2} \mathrm{CH}_{2} \mathrm{CH}_{2} \mathrm{CH}_{3}, 4 \mathrm{H}\right), 1.43$ (s, $\left.\mathrm{CCH}_{3}, 3 \mathrm{H}\right), 1.55$ (m, $\mathrm{NCH}_{2} \mathrm{CH}_{2} \mathrm{CH}_{2} \mathrm{CH}_{3}, 4 \mathrm{H}$ ), 3.90 (t, $J=4.8 \mathrm{~Hz}, \mathrm{NCH}_{2} \mathrm{CH}_{2} \mathrm{CH}_{2} \mathrm{CH}_{3}, 4 \mathrm{H}$ ), 4.55-4.76 (dd, AB, $\left.J=13.6 \mathrm{~Hz}, \mathrm{CCH}_{3} \mathrm{CH}_{2}, 4 \mathrm{H}\right), 6.95(\mathrm{~s}, \mathrm{NCHCHN}, 2 \mathrm{H}), 7.2\left(\mathrm{~m}, \mathrm{C}_{5} \mathrm{H}_{4} \mathrm{~N}, 1 \mathrm{H}\right), 7.4\left(\mathrm{~s}, \mathrm{C}_{5} \mathrm{H}_{4} \mathrm{~N}\right.$, $1 \mathrm{H}), 7.45$ (s, NCHCHN, 2H), $7.65\left(\mathrm{~m}, \mathrm{C}_{5} \mathrm{H}_{4} \mathrm{~N}, 1 \mathrm{H}\right), 8.5$ (d, J=1.2 Hz, $\left.\mathrm{C}_{5} \mathrm{H}_{4} \mathrm{~N}, 1 \mathrm{H}\right) \mathrm{ppm}$. ${ }^{13} \mathrm{C}\left\{{ }^{1} \mathrm{H}\right\}$ NMR (100.6 MHz, DMSO-d $\left.6,293 \mathrm{~K}\right): \delta 14.3,17.6,18.5,31.5,54.6,57.5,63.5$, $120.8,121.3,121.9,122.5,136.4,148.5,158.8,179.4$ ppm.

\section{Synthesis of 2-(1,3-bis(3-propenylimidazolidin-1-yl)-2-methylpropan-2-yl)pyridine} Silver(I) bromide, $6 \mathrm{c}$

This compound was prepared an analogous manner to $\mathbf{6 a}$; from 2-(1,3-bis(3propenylimidazolidin-1-yl)-2-methylpropan-2-yl)pyridine bromide, 5c, (0.51 g, $1 \mathrm{mmol}$ ) and $\mathrm{Ag}_{2} \mathrm{O}(0.27 \mathrm{~g}, 1 \mathrm{mmol})$. The resultant solid was dried in vacuo, giving $6 \mathbf{c}(0.4 \mathrm{~g}, 77$ $\%)$ as a grey creamy powder, which rapidly discoloured on standing. ${ }^{1} \mathrm{H}$ NMR (400.1 
$\left.\mathrm{MHz}, \mathrm{CDCl}_{3}, 293 \mathrm{~K}\right): \delta 1.40\left(\mathrm{~s}, \mathrm{CCH}_{3}, 3 \mathrm{H}\right), 4.6\left(\mathrm{~d}, J=2 \mathrm{~Hz}, \mathrm{NCH}_{2} \mathrm{CHCH}_{2}, 4 \mathrm{H}\right), 4.65-$ 4.75 (dd, $\left.\mathrm{AB}, J=13.2 \mathrm{~Hz}, \mathrm{CCH}_{3} \mathrm{CH}_{2}, 4 \mathrm{H}\right), 5.08$ (d, $\left.J=4 \mathrm{~Hz}, \mathrm{NCH}_{2} \mathrm{CHCH}_{2}, 4 \mathrm{H}\right), 5.7$ (m, $\left.\mathrm{NCH}_{2} \mathrm{CHCH}_{2}, 2 \mathrm{H}\right), 6.26$ (s, NCHCHN, 2H), 6.64 (s, NCHCHN, 2H), 6.95 (d, J=3.2 Hz, $\left.\mathrm{C}_{5} \mathrm{H}_{4} \mathrm{~N}, 1 \mathrm{H}\right), 7.32\left(\mathrm{~m}, \mathrm{C}_{5} \mathrm{H}_{4} \mathrm{~N}, 1 \mathrm{H}\right), 7.5\left(\mathrm{t}, J=0.8 \mathrm{~Hz}, \mathrm{C}_{5} \mathrm{H}_{4} \mathrm{~N}, 1 \mathrm{H}\right), 8.6(\mathrm{~d}, J=1.6 \mathrm{~Hz}$, $\left.\mathrm{C}_{5} \mathrm{H}_{4} \mathrm{~N}, 1 \mathrm{H}\right) \mathrm{ppm} .{ }^{13} \mathrm{C}\left\{{ }^{1} \mathrm{H}\right\} \mathrm{NMR}\left(100.6 \mathrm{MHz}, \mathrm{CDCl}_{3}, 293 \mathrm{~K}\right): \delta 15.2,47.4,59.2,65.8$, $120.4,121.7,122.5,122.9,132.6,132.9,137.5,150.1,160.1,183.6$ ppm.

\section{Synthesis of 2-(2-methyl-1,3-bis(3-propenylimidazolidin-1-yl)propan-2-yl)pyridine} acetonitrile Palladium(II) Chloride, 7

2-(2-methyl-1,3-bis(3-methylimidazolidin-1-yl)propan-2-yl)pyridine Silver(I) bromide 6c $(0.24 \mathrm{~g}, 0.05 \mathrm{mmol})$ was dissolved in $20 \mathrm{~mL} \mathrm{DCM}$, and $\mathrm{PdCl}_{2}\left(\mathrm{CH}_{3} \mathrm{CN}\right)_{2}(129.7 \mathrm{mg}, 0.05$ mmol) was added to the solution of $6 \mathbf{c}$. The mixture was stirred in the dark at room temperature overnight. The solution was filtered to remove $\mathrm{AgBr}$. All volatiles were removed under vacuum. The yellow solid was washed with ether $(2 \times 10 \mathrm{~mL})$ and dried under vacuum. Yield: 75\%, $180 \mathrm{mg}$. ${ }^{1} \mathrm{H}$ NMR (500.1 MHz, DMSO-d 6 , $293 \mathrm{~K}$ ): $\delta 1.15$ (s, $\left.\mathrm{CCH}_{3}, 3 \mathrm{H}\right), 4.22$ (d, $\left.J=14.5 \mathrm{~Hz}, \mathrm{NCH}_{2} \mathrm{CHCH}_{2}, 4 \mathrm{H}\right), 5.12-5.26\left(\mathrm{~m}, \mathrm{CCH}_{3} \mathrm{CH}_{2}, 4 \mathrm{H}\right), 5.57$ (d, $\left.J=14.5 \mathrm{~Hz}, \mathrm{NCH}_{2} \mathrm{CHCH}_{2}, 4 \mathrm{H}\right), 6.15$ (m, $\left.\mathrm{NCH}_{2} \mathrm{CHCH}_{2}, 2 \mathrm{H}\right), 7.21$ (s, NCHCHN, 2H), 7.47 (s, NCHCHN, 2H), $7.4\left(\mathrm{~m}, \mathrm{C}_{5} H_{4} \mathrm{~N}, 1 \mathrm{H}\right), 7.77$ (d, J=8 Hz, $\left.\mathrm{C}_{5} H_{4} \mathrm{~N}, 1 \mathrm{H}\right), 8.01$ (t, $J=6$ $\left.\mathrm{Hz}, \mathrm{C}_{5} H_{4} \mathrm{~N}, 1 \mathrm{H}\right), 8.79\left(\mathrm{~d}, J=3.5 \mathrm{~Hz}, \mathrm{C}_{5} H_{4} \mathrm{~N}, 1 \mathrm{H}\right) \mathrm{ppm} .{ }^{13} \mathrm{C}\left\{{ }^{1} \mathrm{H}\right\} \mathrm{NMR}(125.7 \mathrm{MHz}$, DMSO-d $\left._{6}, 293 \mathrm{~K}\right): \delta 16.4,44.5,51.9,59.6,117.4,118.1,118.6,120.0,125.4,134.1$, 137.6, 148.9, 160.4, 163.1 ppm. $\mathrm{C}_{\mathrm{NHC}}$ not observed. HR-MS (ES), $m / z=486.0851$ [M$\mathrm{Cl}]^{+}$, (calc. for $\mathrm{C}_{21} \mathrm{H}_{25}{ }^{35} \mathrm{ClN}_{5}{ }^{104} \mathrm{Pd}$ : 486.0839).

\section{Synthesis of 2-(2-methyl-1,3-bis(3-methylimidazolidin-1-yl)propan-2-yl)pyridine} Rhodium(I) cyclooctadiene chloride, 8 a

2-(2-methyl-1,3-bis(3-methylimidazolidin-1-yl)propan-2-yl)pyridine bromide, 5a, (0.457 $\mathrm{g}, 1 \mathrm{mmol})$ and $\mathrm{KN}\left(\mathrm{SiMe}_{3}\right)_{2}(0.4 \mathrm{~g}, 1 \mathrm{mmol})$ were placed into a Schlenk tube followed by the addition of THF $(10 \mathrm{~mL})$. The solution was stirred for 2 hours and subsequently filtered into another Schlenk tube containing $10 \mathrm{~mL}$ of a THF solution of $[\mathrm{Rh}(\mathrm{COD}) \mathrm{Cl}]_{2}$ $(0.24 \mathrm{~g}, 0.5 \mathrm{mmol})$. An immediate colour change was observed from light yellow to dark orange and after 5 minutes yellow solid precipitated out. After the reaction mixture was 
stirred at room temperature for $2 \mathrm{~h}$, the volatiles were removed in vacuo. The yellow solid obtained was washed with cold hexane $(2 \times 10 \mathrm{~mL})$ and dried under vacuum. Yield: $65 \%, 164 \mathrm{mg}$. The structure of complex characterised by X-ray diffraction. ${ }^{1} \mathrm{H}$ NMR $\left(500.1 \mathrm{MHz}, \mathrm{CDCl}_{3}, 293 \mathrm{~K}\right): \delta 1.2\left(\mathrm{~s}, \mathrm{CCH}_{3}, 3 \mathrm{H}\right), 2.1-2.6\left(\mathrm{~m}, \mathrm{CHCH}_{2}, 8 \mathrm{H}\right), 3.99$ (s, $\left.\mathrm{NCH}_{3}, 6 \mathrm{H}\right), 4.01$ (d, $\left.J=15 \mathrm{~Hz}, \mathrm{CHCH}_{2}, 2 \mathrm{H}\right), 4.5$ (dd, AB, $\left.J=13.2 \mathrm{~Hz}, \mathrm{CCH}_{3} \mathrm{CH}_{2}, 4 \mathrm{H}\right)$, $5.62\left(\mathrm{~d}, J=24.5 \mathrm{~Hz}, \mathrm{CHCH}_{2}, 2 \mathrm{H}\right), 6.99$ (s, NCHCHN, 2H), 7.02 (s, NCHCHN, 2H), 7.27 $\left(\mathrm{m}, \mathrm{C}_{5} H_{4} \mathrm{~N}, 1 \mathrm{H}\right), 7.64\left(\mathrm{~d}, J=8 \mathrm{~Hz}, \mathrm{C}_{5} H_{4} \mathrm{~N}, 1 \mathrm{H}\right), 7.78\left(\mathrm{t}, J=6 \mathrm{~Hz}, \mathrm{C}_{5} H_{4} \mathrm{~N}, 1 \mathrm{H}\right), 8.58(\mathrm{~d}, J=$ $\left.1 \mathrm{~Hz}, \mathrm{C}_{5} H_{4} \mathrm{~N}, 1 \mathrm{H}\right) \mathrm{ppm} .{ }^{13} \mathrm{C}\left\{{ }^{1} \mathrm{H}\right\} \mathrm{NMR}\left(125.7 \mathrm{MHz}, \mathrm{CDCl}_{3}, 293 \mathrm{~K}\right): \delta 14.2,17.23,27.0$, 29.7, 30.1, 37.1, 45.1, 54.5, 62.6, 64.8, 87.6, 89.2, 118.9, 121.5, 123.7, 127.6, 136.6, 148.1, 162.2, 182.0 (d, $J=52.5 \mathrm{~Hz}$ ) ppm. HR-MS (ES): $m / z=506.1813[\mathrm{M}]^{+}$, (calc. for $\mathrm{C}_{25} \mathrm{H}_{33} \mathrm{~N}_{5} \mathrm{Rh}:$ 506.1791).

\section{Synthesis of 2-(1,3-bis(3-butylimidazolidin-1-yl)-2-methylpropan-2-yl)pyridine}

\section{Rhodium(I) cyclooctadiene Chloride, $8 \mathrm{~b}$}

Complex 8b was prepared from 2-(1,3-bis(3-butylimidazolidin-1-yl)-2-methylpropan-2yl)pyridine bromide, 5b, $(0.54 \mathrm{~g}, 1 \mathrm{mmol}), \mathrm{KN}\left(\mathrm{SiMe}_{3}\right)_{2} \quad(0.4 \mathrm{~g}, 1 \mathrm{mmol})$ and $[\mathrm{Rh}(\mathrm{COD}) \mathrm{Cl}]_{2}(0.24 \mathrm{~g}, 0.5 \mathrm{mmol})$ according to the method described for 8a. A yellow solid was obtained in $75 \%$ yield, $221 \mathrm{mg} .{ }^{1} \mathrm{H}$ NMR (500.1 MHz, $\mathrm{CDCl}_{3}, 293 \mathrm{~K}$ ): $\delta 0.95$ $\left(\mathrm{t}, \quad J=6.2 \mathrm{~Hz}, \quad \mathrm{NCH}_{2} \mathrm{CH}_{2} \mathrm{CH}_{2} \mathrm{CH}_{3}, 6 \mathrm{H}\right), 1.10 \quad\left(\mathrm{~s}, \quad \mathrm{CCH}_{3}, 3 \mathrm{H}\right), 1.40-2.3$ (m, $\mathrm{NCH}_{2} \mathrm{CH}_{2} \mathrm{CH}_{2} \mathrm{CH}_{3}, 8 \mathrm{H}$ and $\mathrm{CHCH}_{2}, 8 \mathrm{H}$ ), 3.77 (m, $\mathrm{NCH}_{2} \mathrm{CH}_{2} \mathrm{CH}_{2} \mathrm{CH}_{3}, 4 \mathrm{H}$ ), 3.97 (s, $\left.\mathrm{CCH}_{3} \mathrm{CH}_{2}, 4 \mathrm{H}\right), 4.04\left(\mathrm{~d}, J=11.5 \mathrm{~Hz}, \mathrm{CHCH}_{2}, 2 \mathrm{H}\right), 5.30$ (d, $\left.J=14 \mathrm{~Hz}, \mathrm{CHCH}_{2}, 2 \mathrm{H}\right), 6.95$ (s, NCHCHN, 2H), $7.15(\mathrm{~s}, \mathrm{NCHCHN}, 2 \mathrm{H}), 7.26\left(\mathrm{~m}, \mathrm{C}_{5} H_{4} \mathrm{~N}, 1 \mathrm{H}\right), 7.70\left(\mathrm{~m}, \mathrm{C}_{5} H_{4} \mathrm{~N}, 1 \mathrm{H}\right)$, $7.75\left(\mathrm{~m}, \mathrm{C}_{5} H_{4} \mathrm{~N}, 1 \mathrm{H}\right), 8.50\left(\mathrm{~d}, J=4.8 \mathrm{~Hz}, \mathrm{C}_{5} H_{4} \mathrm{~N}, 1 \mathrm{H}\right) \mathrm{ppm} .{ }^{13} \mathrm{C}\left\{{ }^{1} \mathrm{H}\right\} \mathrm{NMR}(125.7 \mathrm{MHz}$, $\left.\mathrm{CDCl}_{3}, 293 \mathrm{~K}\right): \delta 13.8,18.8,26.6,30.0,31.7,44.7,49.3,59.5,64.4,66.5,86.8,88.9$, 118.4, 121.7, 123.4, 127.2, 136.4, 147.8, 161.6, 180.9 (d, $J=62.5$ Hz) ppm. HR-MS (ES), $m / z=590.2734[\mathrm{M}]^{+}$(calc. for $\mathrm{C}_{31} \mathrm{H}_{45} \mathrm{~N}_{5} \mathrm{Rh}: 590.2730$ ).

\section{Synthesis of 2-(1,3-bis(3-propenylimidazolidin-1-yl)-2-methylpropan-2-yl)pyridine Rhodium(I) cyclooctadiene Chloride, 8c}

Complex 8c was prepared from 2-(1,3-bis(3-propenylimidazolidin-1-yl)-2-methylpropan2-yl)pyridine bromide, 5c, $(0.51 \mathrm{~g}, 1 \mathrm{mmol}), \mathrm{KN}\left(\mathrm{SiMe}_{3}\right)_{2} \quad(0.4 \mathrm{~g}, 1 \mathrm{mmol})$ and 
$[\mathrm{Rh}(\mathrm{COD}) \mathrm{Cl}]_{2} \quad(0.24 \mathrm{~g}, 0.5 \mathrm{mmol})$ according to the method for 8a. A yellow solid was obtained in $55 \%$ yield, $163 \mathrm{mg} .{ }^{1} \mathrm{H}$ NMR $\left(500.1 \mathrm{MHz}, \mathrm{CDCl}_{3}, 293 \mathrm{~K}\right): \delta 1.34\left(\mathrm{~s}, \mathrm{CCH}_{3}\right.$, $6 \mathrm{H}), 1.63-2.3\left(\mathrm{~m}, \mathrm{CHCH}_{2}, 8 \mathrm{H}\right), 4.2-4.6\left(\mathrm{~m}, \mathrm{CCH}_{3} \mathrm{CH}_{2}, 4 \mathrm{H}, \mathrm{NCH}_{2} \mathrm{CHCH}_{2}, 4 \mathrm{H}\right.$ and $\left.\mathrm{CHCH}_{2}, 2 \mathrm{H}\right), 5.65$ (d, $\left.J=14.2 \mathrm{~Hz}, \mathrm{NCH}_{2} \mathrm{CHCH}_{2}, 4 \mathrm{H}\right), 5.85\left(\mathrm{~m}, \mathrm{CHCH}_{2}, 2 \mathrm{H}\right), 6.90-7-45$ $\left(\mathrm{m}, \mathrm{NCHCHN}, 2 \mathrm{H}, \mathrm{C}_{5} H_{4} \mathrm{~N}, 1 \mathrm{H}, \mathrm{NCHCHN}, 2 \mathrm{H}\right), 7.86\left(\mathrm{~m}, \mathrm{C}_{5} H_{4} \mathrm{~N}, 2 \mathrm{H}\right), 8.59$ (d, J=3.2 $\left.\mathrm{Hz}, \mathrm{C}_{5} H_{4} \mathrm{~N}, 1 \mathrm{H}\right) \mathrm{ppm} .{ }^{13} \mathrm{C}\left\{{ }^{1} \mathrm{H}\right\} \mathrm{NMR}\left(125.7 \mathrm{MHz}, \mathrm{DMSO}_{6}, 293 \mathrm{~K}\right): \delta 13.2,16.6,23.6$, 26.0, 28.5, 44.3, 59.2, 66.0, 87.4, 88.9, 119.4, 122.8, 123.5, 125.8, 126.7, 135.9, 147.0, 161.0, $183.1(\mathrm{~d}, J=75 \mathrm{~Hz}) \mathrm{ppm}$. HR-MS (ES), $m / z=558.2103[\mathrm{M}]^{+}$(calc. for $\mathrm{C}_{29} \mathrm{H}_{37} \mathrm{~N}_{5} \mathrm{Rh}:$ 558.2104).

\section{Synthesis of 2-(2-methyl-1,3-bis(3-methylimidazolidin-1-yl)propan-2-yl)pyridine Iridium(I) cyclooctadiene Chloride, 9a}

2-(2-methyl-1,3-bis(3-methylimidazolidin-1-yl)propan-2-yl)pyridine bromide, 5a, (0.457 $\mathrm{g}, 1 \mathrm{mmol})$ and $\mathrm{KN}\left(\mathrm{SiMe}_{3}\right)_{2}(0.4 \mathrm{~g}, 1 \mathrm{mmol})$ were placed into a Schlenk tube followed by the addition of THF $(10 \mathrm{~mL})$. The solution was stirred for 2 hours and subsequently filtered into another Schlenk tube containing a $10 \mathrm{~mL}$ THF solution of $[\operatorname{Ir}(\mathrm{COD}) \mathrm{Cl}]_{2}$ (335 mg, $0.5 \mathrm{mmol})$. An immediate color change was observed from light orange to dark orange and after half an hour orange solid formed. The reaction mixture was stirred at room temperature for $2 \mathrm{~h}$, and the volatiles were removed in vacuo. The yellow solid obtained was washed with cold hexane $(2 \times 10 \mathrm{~mL})$ and dried under vacuum. Yield: $60 \%$, $178 \mathrm{mg}$. The complex was characterised by X-ray diffraction. ${ }^{1} \mathrm{H}$ NMR $(500.1 \mathrm{MHz}$, $\left.\mathrm{CDCl}_{3}, 293 \mathrm{~K}\right): \delta 1.17$ (s, $\left.\mathrm{CCH}_{3}, 3 \mathrm{H}\right), 1.9-2.4\left(\mathrm{~m}, \mathrm{CHCH}_{2}, 8 \mathrm{H}\right), 3.36\left(\mathrm{~s}, \mathrm{NCH}_{3}, 6 \mathrm{H}\right), 3.95$ $\left(\mathrm{d}, J=4.5 \mathrm{~Hz}, \mathrm{CHCH}_{2}, 2 \mathrm{H}\right), 4.1\left(\mathrm{~s}, \mathrm{CCH}_{3} \mathrm{CH}_{2}, 4 \mathrm{H}\right), 5.4\left(\mathrm{~d}, J=14.5 \mathrm{~Hz}, \mathrm{CHCH}_{2}, 2 \mathrm{H}\right), 7.02$ (s, NCHCHN, 2H), 7.07 (s, NCHCHN, 2H), $7.27\left(\mathrm{~m}, \mathrm{C}_{5} H_{4} \mathrm{~N}, 1 \mathrm{H}\right), 7.61(\mathrm{~d}, J=8 \mathrm{~Hz}$, $\left.\mathrm{C}_{5} H_{4} \mathrm{~N}, 1 \mathrm{H}\right), 7.77\left(\mathrm{t}, J=7.5 \mathrm{~Hz}, \mathrm{C}_{5} H_{4} \mathrm{~N}, 1 \mathrm{H}\right), 8.58\left(\mathrm{~d}, J=1 \mathrm{~Hz}, \mathrm{C}_{5} H_{4} \mathrm{~N}, 1 \mathrm{H}\right) \mathrm{ppm} .{ }^{13} \mathrm{C}\left\{{ }^{1} \mathrm{H}\right\}$ NMR (125.7 MHz, $\left.\mathrm{CDCl}_{3}, 293 \mathrm{~K}\right), \delta$ 17.1, 24.6, 27.0, 30.3, 36.9, 45.7, 59.6, 67.0, 74.9, 76.6, 118.9, 121.2, 121.6, 123.4, 127.7, 136.7, 148.1, 162.2, 177.8 ppm. HR-MS (ES), $m / z=594.2350[\mathrm{M}]^{+}$(calc. for $\mathrm{C}_{25} \mathrm{H}_{33} \mathrm{~N}_{5}$ Ir: 594.2342). 


\section{Synthesis of 2-(1,3-bis(3-butylimidazolidin-1-yl)-2-methylpropan-2-yl)pyridine}

Iridium(I) cyclooctadiene bromide, $9 \mathrm{~b}$

Complex 9b was prepared from 2-(1,3-bis(3-butylimidazolidin-1-yl)-2-methylpropan-2yl)pyridine bromide, $\mathbf{5 b},(0.54 \mathrm{~g}, 1 \mathrm{mmol}), \mathrm{KN}\left(\mathrm{SiMe}_{3}\right)_{2} \quad(0.4 \mathrm{~g}, 1 \mathrm{mmol})$ and $[\operatorname{Ir}(\mathrm{COD}) \mathrm{Cl}]_{2} \quad(0.33 \mathrm{~g}, 0.5 \mathrm{mmol})$ according to the method of 9a. Orange solid was obtained in $60 \%$ yield, $200 \mathrm{mg}$. The complex was characterised by X-ray diffraction. ${ }^{1} \mathrm{H}$ NMR (400.1 MHz, $\mathrm{CDCl}_{3}, 293 \mathrm{~K}$ ): $\delta 0.99$ (t, $J=6 \mathrm{~Hz}, \mathrm{NCH}_{2} \mathrm{CH}_{2} \mathrm{CH}_{2} \mathrm{CH}_{3}, 6 \mathrm{H}$ ), 1.15 (s, $\left.\mathrm{CCH}_{3}, 3 \mathrm{H}\right), 1.45-2.4$ (m, $\mathrm{NCH}_{2} \mathrm{CH}_{2} \mathrm{CH}_{2} \mathrm{CH}_{3}, 8 \mathrm{H}$ and $\left.\mathrm{CHCH}_{2}, 8 \mathrm{H}\right), 3.87$ (m, $\left.\mathrm{NCH}_{2} \mathrm{CH}_{2} \mathrm{CH}_{2} \mathrm{CH}_{3}, 4 \mathrm{H}\right), 4.05$ (s, $\left.\mathrm{CCH}_{3} \mathrm{CH}_{2}, 4 \mathrm{H}\right), 4.1$ (d, $\left.\mathrm{J}=12 \mathrm{~Hz}, \mathrm{CHCH}_{2}, 2 \mathrm{H}\right), 5.36$ (d, $\left.J=14 \mathrm{~Hz}, \mathrm{CHCH}_{2}, 2 \mathrm{H}\right), 7.0(\mathrm{~s}, \mathrm{NCHCHN}, 2 \mathrm{H}), 7.22(\mathrm{~s}, \mathrm{NCHCHN}, 2 \mathrm{H}), 7.27\left(\mathrm{~m}, \mathrm{C}_{5} H_{4} \mathrm{~N}\right.$, $1 \mathrm{H}), 7.78\left(\mathrm{~m}, \mathrm{C}_{5} H_{4} \mathrm{~N}, 1 \mathrm{H}\right), 7.8\left(\mathrm{~m}, \mathrm{C}_{5} H_{4} \mathrm{~N}, 1 \mathrm{H}\right), 8.55\left(\mathrm{~d}, J=4.4 \mathrm{~Hz}, \mathrm{C}_{5} H_{4} \mathrm{~N}, 1 \mathrm{H}\right) \mathrm{ppm}$. ${ }^{13} \mathrm{C}\left\{{ }^{1} \mathrm{H}\right\}$ NMR $\left(100.6 \mathrm{MHz}, \mathrm{CDCl}_{3}, 293 \mathrm{~K}\right): \delta 14.1,18.7,20.6,30.1,31.6,31.8,33.5$, 47.1, 50.7, 61.0, 75.6, 77.7, 120.0, 120.9, 123.0, 125.7, 138.2, 149.3, 163.6, 178.2 ppm. HR-MS (ES), $m / z=680.3282[\mathrm{M}]^{+}$(calc. for $\mathrm{C}_{31} \mathrm{H}_{45} \mathrm{~N}_{5} \mathrm{Ir}$ : 680.3304).

\section{Synthesis of 2-(1,3-bis(3-propenylimidazolidin-1-yl)-2-methylpropan-2-yl)pyridine}

\section{Iridium(I) cyclooctadiene chloride, 9c}

Complex 9c was prepared from 2-(1,3-bis(3-propenylimidazolidin-1-yl)-2-methylpropan2-yl)pyridine bromide, 5c, $(0.51 \mathrm{~g}, 1 \mathrm{mmol}), \mathrm{KN}\left(\mathrm{SiMe}_{3}\right)_{2} \quad(0.4 \mathrm{~g}, 1 \mathrm{mmol})$ and $[\mathrm{Ir}(\mathrm{COD}) \mathrm{Cl}]_{2} \quad(0.33 \mathrm{~g}, 0.5 \mathrm{mmol})$ according to the general method for 9a. Orange solid was obtained in $55 \%$ yield, $180 \mathrm{mg} .{ }^{1} \mathrm{H} \mathrm{NMR}\left(500.1 \mathrm{MHz}, \mathrm{CDCl}_{3}, 293 \mathrm{~K}\right.$ ): $\delta 1.32$ (s, $\left.\mathrm{CCH}_{3}, 6 \mathrm{H}\right), 1.63-2.3\left(\mathrm{~m}, \mathrm{CHCH}_{2}, 8 \mathrm{H}\right), 4.0-4.22\left(\mathrm{~m}, \mathrm{CCH}_{3} \mathrm{CH}_{2}, 4 \mathrm{H}\right.$ and $\mathrm{NCH}_{2} \mathrm{CHCH}_{2}$, $4 \mathrm{H}), 4.26\left(\mathrm{~d}, J=14.4 \mathrm{~Hz}, \mathrm{NCH}_{2} \mathrm{CHCH}_{2}, 2 \mathrm{H}\right), 5.34$ (d, $\left.J=14.4 \mathrm{~Hz}, \mathrm{NCH}_{2} \mathrm{CHCH}_{2}, 2 \mathrm{H}\right)$, 5.57 (m, $\left.\mathrm{CHCH}_{2}, 2 \mathrm{H}\right), 6.94$ (d, J=8 Hz, $\left.\mathrm{CHCH}_{2}, 2 \mathrm{H}\right), 7.0$ (s, $\left.\mathrm{NCHCHN}, 2 \mathrm{H}\right), 7.27$ (m, $\left.\mathrm{C}_{5} H_{4} \mathrm{~N}, 1 \mathrm{H}\right), 7.44(\mathrm{~s}, \mathrm{NCHCHN}, 2 \mathrm{H}), 7.82\left(\mathrm{~m}, \mathrm{C}_{5} H_{4} \mathrm{~N}, 2 \mathrm{H}\right), 8.52\left(\mathrm{~d}, J=3.2 \mathrm{~Hz}, \mathrm{C}_{5} H_{4} \mathrm{~N}\right.$, 1H) ppm. ${ }^{13} \mathrm{C}\left\{{ }^{1} \mathrm{H}\right\}\left(125.7 \mathrm{MHz}, \mathrm{DMSO}-\mathrm{d}_{6}\right): \delta 11.6,17.4,27.0,30.2,45.9,59.9,75.6$, 75.7, 77.2, 118.8, 119.8, 121.6, 123.6, 125.7, 137.0, 147.7, 162.0, 179.4 ppm. HR-MS (ES), $m / z=648.2676[\mathrm{M}]^{+}$(calc. for $\mathrm{C}_{31} \mathrm{H}_{45} \mathrm{~N}_{5} \mathrm{Ir}: 648.2678$ ). 


\section{Catalytic Transfer Hydrogenation}

The $\mathrm{Ir} / \mathrm{Rh}$ catalyst precursor $(1,0.3,0.01 \mathrm{~mol} \%)$ was dissolved in a solution of $\mathrm{K}^{t} \mathrm{BuO}$ $(10 \mathrm{mmol})$ in 2-propanol $(5 \mathrm{~mL})$ and substrate $(1 \mathrm{mmol})$ in a Schlenk tube. The solution was heated to $80^{\circ} \mathrm{C}$ for 30 minutes, the final conversion determined by ${ }^{1} \mathrm{H}$ NMR.

Reaction progress was monitored by ${ }^{1} \mathrm{H}$ NMR and GC-MS: aliquots $(0.1 \mathrm{ml})$ were taken every 3 or 5 minutes for the first hour, and finally another two samples were taken after 2 hours. The samples were filtered through a short pad of silica, and the silica was washed with DCM $(2 \times 2 \mathrm{ml})$, and the sample subsequently analysed. Yields and substrate identities were determined by GC-MS analysis of reaction mixtures using an Agilent Technologies 6890N GC system with Agilent Technologies 5973 inert MS detector with MSD. Column: Agilent 190915-433 capillary, $0.25 \mathrm{~mm}$ x $30 \mathrm{~m}$ x $0.25 \mu \mathrm{m}$. Initial temperature $50{ }^{\circ} \mathrm{C}$, held for 4 minutes, ramp $5{ }^{\circ} \mathrm{C} /$ minute next $100{ }^{\circ} \mathrm{C}$, ramp $10{ }^{\circ} \mathrm{C} /$ minute next $240{ }^{\circ} \mathrm{C}$ hold for 15 minutes. The temperature of the injector and detector were held at $240{ }^{\circ} \mathrm{C}$. The retention times for analytes (minutes), e.g. 4-bromoacetophenone 18.3 and 1-(4-bromophenyl)ethanol 18.7.

\section{Crystallographic studies}

Crystal data and details of the structure determinations are listed in Table 3. Intensity data were collected at low temperature with an Enraf-Nonius Kappa CCD diffractometer. Data were corrected for air and detector absorption, Lorentz and polarisation effects, absorption by the crystal was treated with a semiempirical multi-scan method. The structures were solved by direct methods and refined by full-matrix least squares methods based on $F^{2}$ against all unique reflections (8a). Owing to unsatisfactory refinement, the structure of 9b was refined against all data with $\mathrm{I}>-5 \sigma(\mathrm{I})$. All non-hydrogen atoms were given anisotropic displacement parameters. Hydrogen atoms were input at calculated positions and were refined with the riding model.

Table 3 here 


\section{Acknowledgements}

We would like to thank the EPSRC and TÜBİTAK, Turkey (DB2219, postdoctoral fellowship for SY) for funding this work. We would also like to thanks Johnson Matthey for the loan of precious metals.

\section{References}

1. (a) P. Chaudhuri and K. Wieghardt, Prog. Inorg. Chem., 1987, 35, 329; (b)

2. (a) S. Trofimenko, Chem. Rev., 1993, 93, 943; (b) S. Trofimenko, The Coordination Chemistry of Pyrazole-Derived Ligands, ed. S. J. Lippard, Wiley, New York, 1986; (c) S. Trofimenko, Chem. Rev., 1972, 72, 497; (d) S. Trofimenko, Scorpionates: the Coordination Chemistry of Polypyrazolylborate Ligands, Imperial College Press, London, 198; (e) C. Pettinari and C. Santini, in Comprehensive Coordination Chemistry II, ed. J. A. McCleverty and T. J. Meyer, Elsevier, Oxford, 2004, vol. 1, p. 159; (f) S. Trofimenko, Polyhedron, 2004, 23, 197; (g) A. Otero, J. Fernández-Baeza, A. Antiñolo, J. Tejeda and A. LaraSanchez, Dalton Trans., 2004, 1499.

3. (a) D. L. Reger, Comments Inorg. Chem., 1999, 21, 1; (b) H. Bigmore, S. C. Lawrence, P. Mountford and C. S. Tredget, Dalton Trans., 2005, 635.

4. S. Friedrich, M. Schubart, L. H. Gade, I. J. Scowen and M. McPartlin, Chem. Ber. / Recueil, 1997, 130, 1751.

5. F. G. N. Cloke, P. B. Hitchcock and J. B. Love, J. Chem. Soc., Dalton Trans., $1995,25$.

6. (a) S. A. Harris, J. T. Ciszewski and A. L. Odom, Inorg. Chem., 2001, 40, 1987;

(b) C. Cao, J. T. Ciszewski and A. L. Odom, Organometallics, 2001, 20, 5011.

7. T. A. Lowes, B. D. Ward, R. A. Whannel, S. R. Dubberley and P. Mountford, Chem. Commun., 2005, 113.

8. M. E. G. Skinner, Y. Li and P. Mountford, Inorg. Chem., 2002, 41, 1110.

9. B. D. Ward, A. Maisse-François, P. Mountford and L. H. Gade, Chem. Commun., $2004,704$. 
10. a) E. Mas-Marzá, M. Poyatos, M. Sanaú and E. Peris, Organometallics, 2004, 23, 323; b) E. Mas-Marzá, M. Sanaú and E. Peris, J. Organomet. Chem., 2005, 690, 5576; c) F. Ekkehardt Hahn, C. Holtgrewe, T. Pape, M. Martin, E. Sola, L. A. Oro, Organometallics, 2005, 24, 2203

11. for example; a) W. A. Herrmann, C. Köcher, Angew. Chem. Int. Ed. Engl. 1997, 36, 2162; b) D. Bourissou, O. Guerret, F. P. Gabbaï, G. Bertrand, Chem. Rev. 2000, 100, 39; c) W. A. Herrmann, Angew. Chem. Int. Ed. Engl. 2002, 41, 1290; d) F. K. Zinn, M. S. Viciu, S. P. Nolan, Annu. Rep. Prog. Chem., Sect. B. 2004, 100, 231; e) K. J. Cavell, D. S. McGuinness. Coord. Chem. Revs. 2004, 248, 671; f) E. Peris, E. Crabtree, Coord. Chem. Revs. 2004, 248, 2239; g) C. M. Crudden, D. P. Allen, Coord. Chem. Revs. 2004, 248, 2247; h) V. César, S. BelleminLaponnaz, L. H. Gade, Chem. Soc. Rev., 2004, 33, 619; i) N-Heterocyclic Carbenes in Synthesis (Ed.: S. P. Nolan), Wiley-VCH, Weinheim, 2006; pp. 73102; j) K. J. Cavell, D. S. McGuinness, Palladium Complexes with Carbonyl, Isocyanide and Carbene Ligands. In Comprehensive Organometallic Chemistry III (Eds.: R. H. Crabtree, D. M. P. Mingos, A. J. Canty), Elsevier, 2007, vol. 8, pp. 197-268; k) D. Pugh, A. A. Danopoulos, Coord. Chem. Rev. 2007, 251, 610; 1) R. E. Douthwaite, Coord. Chem. Rev. 2007, 251, 702; m) L. H. Gade, S. Bellemin-Laponnaz, Coord. Chem. Rev. 2007, 251, 718; n) J. A. Mata, M. Poyatos, E. Peris, Coord. Chem. Rev. 2007, 251, 841; o) W. J. Sommer, M. Weck, Coord. Chem. Rev. 2007, 251, 860; 873; p) F. E. Hahn, M. C. Jahnke, Angew. Chem. Int. Ed. 2008, 47, 3122; q) O. Kaufhold, F. E. Hahn, Angew. Chem. Int. Ed., 2008, 47, 4057; (r) A. T. Norman, K. J. Cavell, Eur. J. Inorg. Chem. 2008, 2781; s) K. J. Cavell, Dalton Trans. 2008, 6676; K. J. Cavell, Dalton Trans. 2008, 6676; p) O. H. Winkelmann, A. Riekstins, S. P. Nolan, O. Navarro, Organometallics 2009, 28, 5809

12. for example: a) V. Lavallo, Y. Canac, C. Prasang, B. Donnadieu and G. Bertrand, Angew. Chem., Int Ed., 2005, 44, 5705; b) V. Lavallo, Y. Canac, A. Dehope, B. Donnadie and G. Bertrand, Angew. Chem., Int. Ed., 2005, 44, 7236; c) D. Martin, A. Baceiredo, H. Gornitzka, W. W. Schoeller and G. Bertrand, Angew. Chem., Int. Ed., 2005, 44, 1700; d) V. Lavallo Y. Canac, B. Donnadieu, W. W. Schoeller and 
G. Bertrand, Angew Chem., Int. Ed., 2006, 45, 3488; e) V. Lavallo, Y. Canac, B. Donnadieu, W. W. Schoeller and G. Bertrand, Science, 2006, 312, 722R. f) Jazzar, J.-B. Bourg, R. D Dewhurst, B. Donnadieu and G. Bertrand, J. Org. Chem., 2007, 72, 3492; g) G. D. Frey, V. Lavallo, B. Donnadieu, W. W. Schoeller and G. Bertrand, Science, 2007, 316, 439

13. a) C. C. Scarborough, M. J. W. Grady, I. A. Guzei, B. A. Gandhi, E. E. Bunel, S. S. Stahl, Angew. Chem. Int. Ed. 2005, 44, 5269; b) C. C. Scarborough, B. V. Popp, I. A. Guzei, S. S. Stahl, J. Organomet. Chem. 2005, 690, 6143; c) M. M. Rogers, J. E. Wendlandt, I. A. Guzei, S. S. Stahl, Org. Lett. 2006, 8, 2257; d) P. Bazinet, T.-G. Ong, J. S. O’Brien, N. Lavoie, E. Bell, G. P. A. Yap, I. Korobkov, D. S. Richeson, Organometallics 2007, 26, 2885; e) V. Cesar, N. Lugan, G. Lavigne, J. Am. Chem. Soc. 2008, 130, 11286; f) D. M. Khramov, E. L. Rosen, V. M. Lynch, C. W. Bielawski, Angew. Chem. Int. Ed. 2008, 47, 2267; g) T. W. Hudnall, C. W. Bielawski, J. Am. Chem. Soc. 2009, 131, 16039; h) U. Siemeling, C. Färber, M. Leibold, C. Bruhn, P. Mucke, R. F. Winter, B. Sarkar, M. Von Hopffgarten, G. Frenking, Eur. J. Inorg. Chem. 2009, 4607; i) M. Iglesias, D. J. Beetstra, A. Stasch, P. N. Horton, M. B. Hursthouse, S. J. Coles, K. J. Cavell, A. Dervisi, I. A. Fallis, Organometallics, 2007, 26, 4800; j) M. Iglesias, D. J. Beetstra, J. C. Knight, L-L. Ooi, A. Stasch, S. J. Coles, L. Male, M. B. Hursthouse, K. J. Cavell, A. Dervisi, I. A. Fallis, Organometallics, 2008, 27, 3279; k) Iglesias, M.; Beetstra, D. J.; B. Kariuki, K. J. Cavell, A. Dervisi, I. A. Fallis, Eur. J. Inorg. Chem. 2009, 13, 1913; 1) A. Binobaid, M. Iglesias, D. J. Beetstra, B. Kariuki, A. Dervisi, I. A. Fallis, I. A., K. J. Cavell, Dalton Trans 2009, 7099; m) E. L. Kolychev, I. A. Portnyagin, V. V. Shuntikov, V. N. Khrustalev, M. S. Nechaev, J. Organomet. Chem. 2009, 694, 2454; n) M. Iglesias, D. J. Beetstra, K. J. Cavell, A. Dervisi, I. A. Fallis, B. Kariuki, R. W. Harrington, W. Clegg, P. N. Horton, S. J. Coles, M. B. Hursthouse, Eur. J. Inorg. Chem. 2010, 1604; o) P. D. Newman, K. J. Cavell, B. M. Kariuki, Organometallics, 2010, 29, 2724. 
14. a) F. H. Allen and O. Kennard, Chemical Design Automation News, 1993, 8, 1 \& 31; b) D. A. Fletcher, R. F. McMeeking and D. J. Parkin, Chem. Inf. Comput. Sci., 1996, 36, 746 .

15. M. Albrecht, J. R. Miecznikowski, A. Samuel, J. W. Faller and R. H. Crabtree, Organometallics, 2002, 21, 3596

16. S. C. Zinner, C. F. Rentzsch, E. Herdtweck, W. A. Herrmann, F. E. Kühn, Dalton Trans. 2009, 7055

17. a) H. Türkmen, T. Pape, F. E. Hahn, B. Çetinkaya, Organometallics 2008, 27, 571; b) H. Türkmen, T. Pape, F. E. Hahn, B. Çetinkaya, Eur. J. Inorg. Chem. 2008, 5418

18. E. Mas-Marza, M. Sanau, E. Peris, Inorg. Chem. 2005, 44, 9961

19. J.-F. Sun, F. Chen, B. A. Dougan, H.-J. Xu, Y. Cheng, Y.-Z. Li, X.-T. Chen, Z.-L. Xue, J. Organomet. Chem. 2009, 694, 2096

20. Abeer Binobaid, Manuel Iglesias, Dirk Beetstra, Athanasia Dervisi, Ian Fallis, and Kingsley J. Cavell, Eur. J. Inorg. Chem., 2010, published on web 22/10/2010

21. G. Giordano and R. H. Crabtree, Inorg. Synth., 1990, 28, 88. 
Table 1: Selected bond lengths $(\AA)$ and angles $\left(^{\circ}\right)$ for $\mathbf{8 a}$ and $\mathbf{9 b}$. Values in parentheses are for the crystallographically independent molecule.

\begin{tabular}{ccc}
\hline & $\mathbf{8 a}$ & $\mathbf{9 b}$ \\
\hline $\mathrm{M}(1)-\mathrm{C}(1)$ & $2.163(12)$ & $2.168(9)[2.189(10)]$ \\
$\mathrm{M}(1)-\mathrm{C}(2)$ & $2.036(11)$ & $2.185(9)[2.186(9)]$ \\
$\mathrm{M}(1)-\mathrm{C}(5)$ & $2.170(12)$ & $2.182(9)[2.177(10)]$ \\
$\mathrm{M}(1)-\mathrm{C}(6)$ & $2.240(13)$ & $2.185(9)[2.178(9)]$ \\
$\mathrm{M}(1)-\mathrm{C}(17)$ & $1.998(12)$ & $2.033(9)[2.027(9)]$ \\
$\mathrm{M}(1)-\mathrm{C}(21)$ & $1.916(12)$ & $2.059(9)[2.047(9)]$ \\
$\mathrm{N}(2)-\mathrm{C}(17)-\mathrm{N}(3)$ & $106.7(9)$ & $104.2(8)[104.9(7)]$ \\
$\mathrm{N}(4)-\mathrm{C}(21)-\mathrm{N}(5)$ & $109.6(9)$ & $104.7(7)[104.7(7)]$ \\
$\mathrm{C}(17)-\mathrm{M}(1)-\mathrm{C}(21)$ & $85.1(4)$ & $87.1(3)[84.7(3)]$ \\
\hline
\end{tabular}


Table 2. Results of Hydrogen Transfer Reactions

\begin{tabular}{|c|c|c|c|c|c|}
\hline Entry & Catalyst & Substrate (1 mmol) & $\begin{array}{c}\text { Catalyst } \\
\text { Concentration } \\
(\mathbf{m o l} \%)\end{array}$ & $\begin{array}{l}\text { Time } \\
\text { (min) }\end{array}$ & $\begin{array}{c}\text { Conversion } \\
\%\end{array}$ \\
\hline 1 & $9 c$ & $p$-bromoacetophenone & 0.3 & 30 & 100 \\
\hline 2 & $9 c$ & “ & 0.01 & 30 & 60 \\
\hline 3 & $9 b$ & "، & 0.3 & 30 & 100 \\
\hline 4 & $9 b$ & ، & 0.01 & 30 & 55 \\
\hline 5 & $9 \mathbf{a}$ & ، & 0.3 & 30 & 100 \\
\hline 6 & $9 \mathbf{a}$ & ، & 0.01 & 30 & 45 \\
\hline 7 & $9 c$ & $p$-methylacetophenone & 0.3 & 60 & 100 \\
\hline 8 & $9 \mathrm{c}$ & “ & 0.01 & 60 & 50 \\
\hline 9 & $9 b$ & “ & 0.3 & 60 & 100 \\
\hline 10 & $9 b$ & 6 & 0.01 & 60 & 46 \\
\hline 11 & $9 \mathbf{a}$ & " & 0.3 & 60 & 80 \\
\hline 12 & $9 \mathbf{a}$ & ، & 0.01 & 60 & 30 \\
\hline 13 & $9 c$ & cyclohexanone & 0.3 & 30 & 100 \\
\hline 14 & $9 c$ & ، & 0.01 & 30 & 100 \\
\hline 15 & $9 b$ & ، & 0.3 & 30 & 100 \\
\hline 16 & $9 b$ & “" & 0.01 & 30 & 100 \\
\hline 17 & $9 \mathbf{a}$ & " & 0.3 & 30 & 100 \\
\hline 18 & $9 \mathbf{a}$ & “" & 0.01 & 30 & 90 \\
\hline 19 & $8 \mathbf{a}$ & $p$-bromoacetophenone & 0.3 & 16 hours & 100 \\
\hline
\end{tabular}


Table 3 Details of the crystal structure determinations for $\mathbf{8 a}$ and $\mathbf{9 b}$

\begin{tabular}{|c|c|c|}
\hline & $8 \mathbf{8}$ & $9 \mathbf{b}$ \\
\hline Empirical formula & $\mathrm{C}_{25} \mathrm{H}_{33} \mathrm{ClN}_{5} \mathrm{Rh}$ & $\mathrm{C}_{31} \mathrm{H}_{45} \mathrm{BrIrN}_{5}$ \\
\hline$M_{r}$ & 541.93 & 759.86 \\
\hline$T / \mathrm{K}$ & 150 & 150 \\
\hline$\lambda / \AA$ & 0.71073 & 0.71073 \\
\hline Crystal system & Monoclinic & Triclinic \\
\hline Space group & $\mathrm{P} 2{ }_{1} / \mathrm{a}$ & P-1 \\
\hline$a / \AA$ & $14.885(3)$ & $14.109(3)$ \\
\hline$b / \AA$ & $13.167(3)$ & $15.263(3)$ \\
\hline$c / \AA ̊$ & $13.902(3)$ & $16.214(3)$ \\
\hline$\alpha /^{\circ}$ & 90 & $117.13(3)$ \\
\hline$\beta /^{\circ}$ & $115.41(3)$ & $90.52(3)$ \\
\hline$\gamma /{ }^{\circ}$ & 90 & $95.31(3)$ \\
\hline$V / \AA^{3}$ & $2461.1(11)$ & $3088.9(14)$ \\
\hline$Z$ & 4 & 4 \\
\hline$D_{c} / \mathrm{mg} \mathrm{m}^{-3}$ & 1.462 & 1.634 \\
\hline$\mu(\mathrm{MoK} \alpha) / \mathrm{mm}^{-1}$ & 0.825 & 5.644 \\
\hline$F_{000}$ & 1120 & 1512 \\
\hline Crystal dimensions/mm & $0.10 \times 0.10 \times 0.05$ & $0.10 \times 0.10 \times 0.05$ \\
\hline$\theta$ range $/^{\circ}$ & $8-21$ & $4-26$ \\
\hline \multirow[t]{3}{*}{ Index ranges $h, k, l$} & $-13 \leq h \leq 12$ & $-17 \leq h \leq 17$ \\
\hline & $0 \leq k \leq 12$ & $-19 \leq k \leq 16$ \\
\hline & $0 \leq l \leq 14$ & $0 \leq l \leq 20$ \\
\hline Reflections measured & 3401 & 17730 \\
\hline Unique reflections $\left[R_{\text {int }}\right]$ & $2026[0.054]$ & $12416[0.058]$ \\
\hline Max, min trans. & $0.96,0.92$ & $0.75,0.57$ \\
\hline Data/restraints/parameters & 2024 / 0 / 289 & $12379 / 42$ / 685 \\
\hline GOF on $F^{2}$ & 1.1528 & 0.9728 \\
\hline $\begin{array}{l}\mathrm{R} \text { indices }[F>4 \sigma(F)] \mathrm{R}(F), \\
\mathrm{wR}\left(F^{2}\right)\end{array}$ & $0.074,0.134$ & $0.062,0.133$ \\
\hline $\begin{array}{l}\mathrm{R} \text { indices (all data) } \mathrm{R}(F) \text {, } \\
\mathrm{wR}\left(F^{2}\right)\end{array}$ & $0.093,0.141$ & $0.089,0.146$ \\
\hline Largest residual peak/e $\AA^{-3}$ & $0.74,-0.84$ & $2.31,-2.10$ \\
\hline
\end{tabular}


<smiles>[X]C12N([SiH3])CC(C)(c3cccn3C1([X])[X])N2[SiH3]</smiles>

I<smiles>[X]C([X])([X])N1CCCN([Si])C1([X])[X]</smiles>

IV

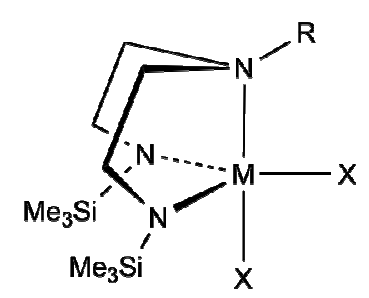

II

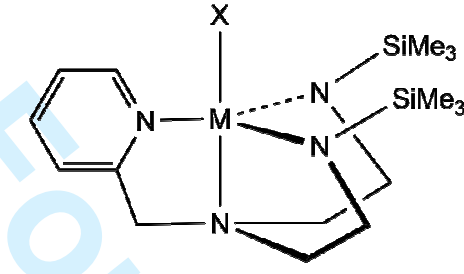

V<smiles>[X]C1(C)CC2(CN(C)CC2([X])[X])CN1C</smiles>

III

Scheme 1: Selected amido- and NHC-based scorpionate ligands

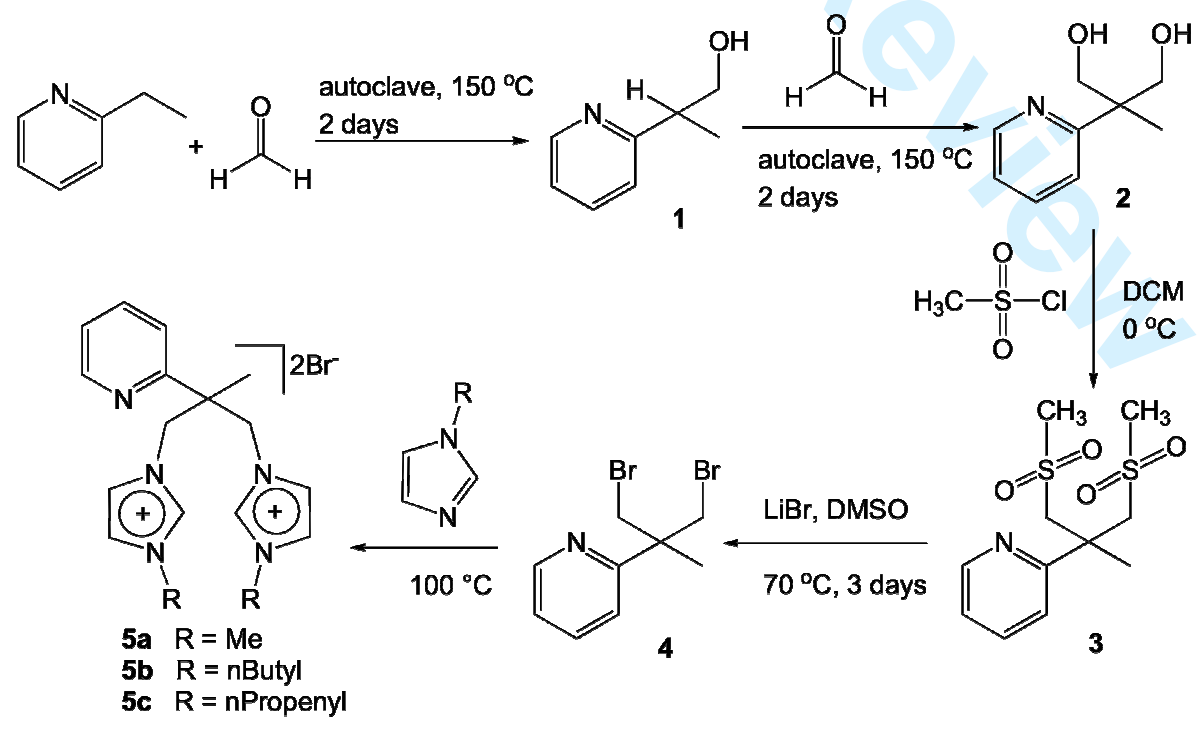

Scheme 2: Synthesis of the bis-imidazolium ligand precursors 
Scheme 3: Synthesis of the Ag-bis-NHC complexes 6a-c

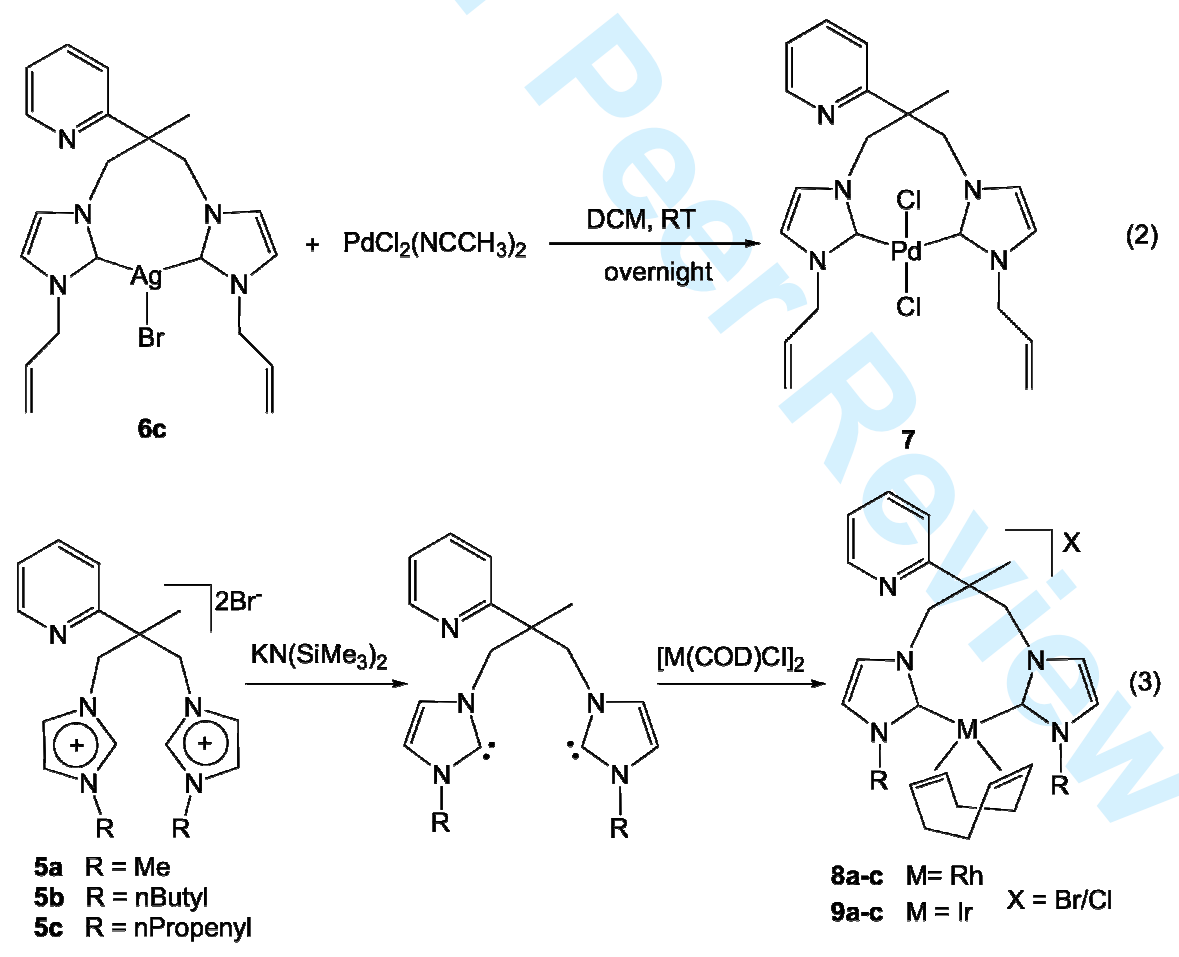

Scheme 4: Synthesis of transition metal complexes of the bis-imidazole ligands 


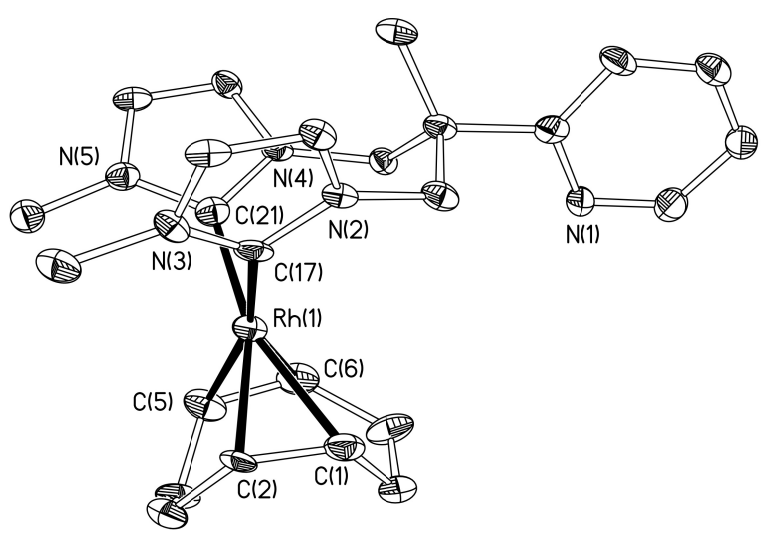

Figure 1: Molecular structure of $\left[\mathrm{Rh}\left(\mathrm{NC}_{2}{ }^{\mathrm{Me}}\right)(\mathrm{COD})\right] \mathrm{Cl}(\mathbf{8 a}) . \mathrm{H}$ atoms and anion omitted for clarity, thermal ellipsoids are drawn at $25 \%$ probability

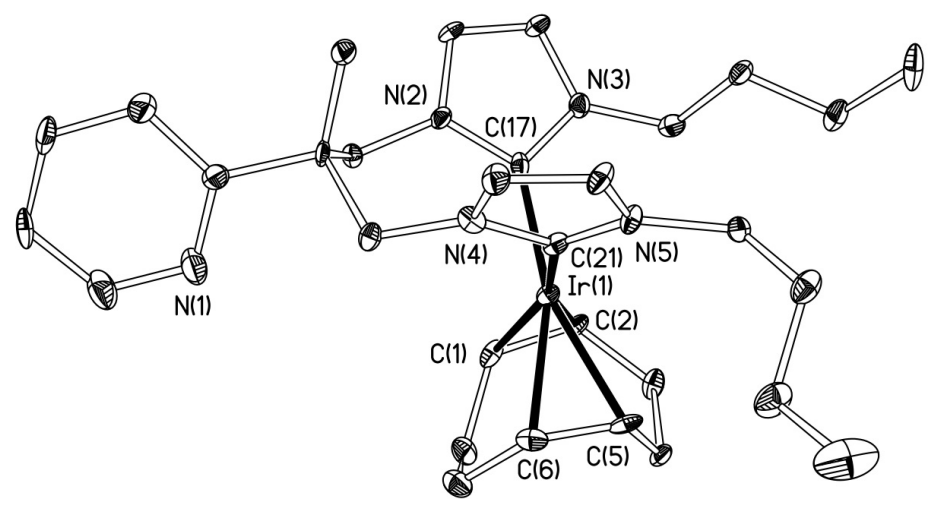

Figure 2: Molecular structure of $\left[\operatorname{Ir}\left(\mathrm{NC}_{2}{ }^{\mathrm{Bu}}\right)(\mathrm{COD})\right] \mathrm{Br}(\mathbf{9 b})$. H atoms, the second crystallographically independent molecule, and anion omitted for clarity, thermal ellipsoids are drawn at $25 \%$ probability<smiles>[R]c1ccc(C(C)=O)cc1</smiles> 


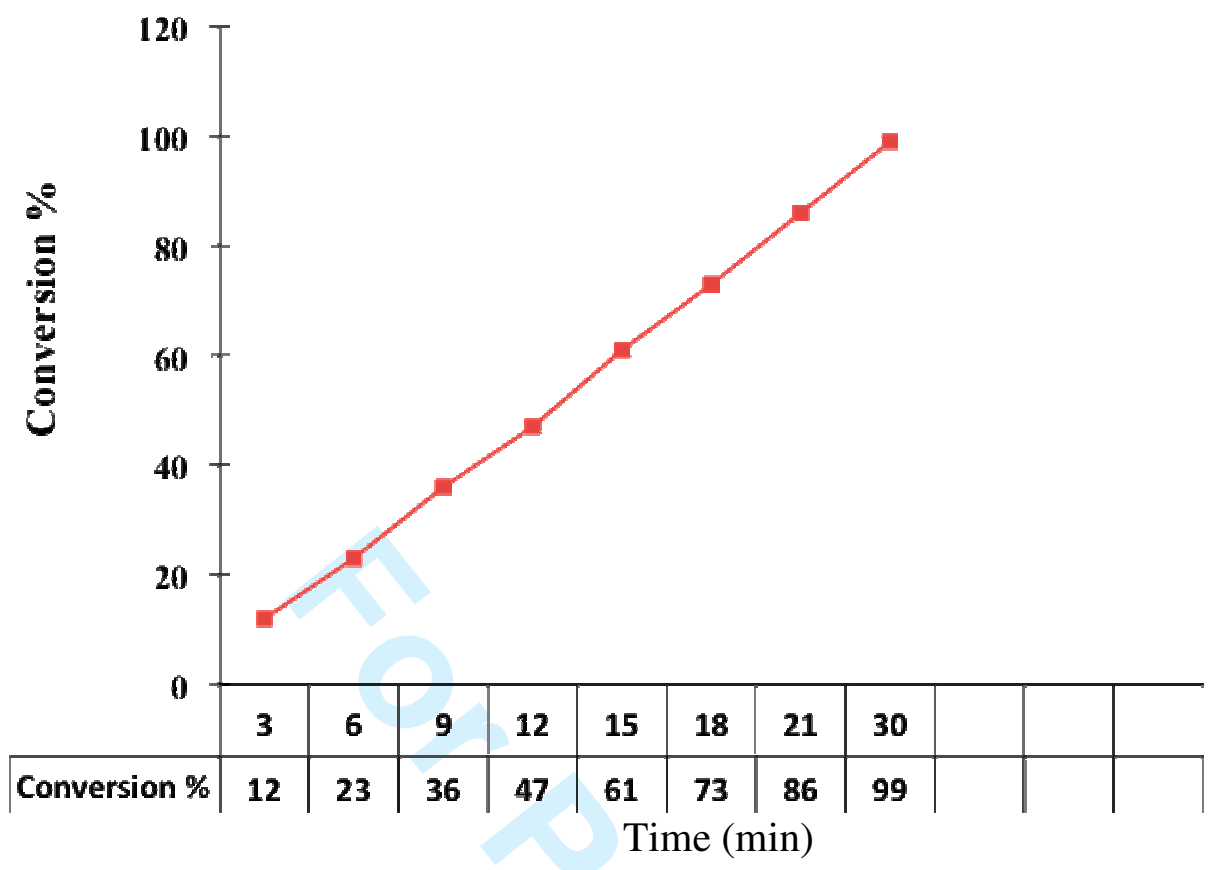

Figure 3: Plot of conversion versus time for $p$-bromoacetophenone (1 mmol) catalysed by $9 \mathbf{c}(0.3 \mathrm{~mol} \%)$

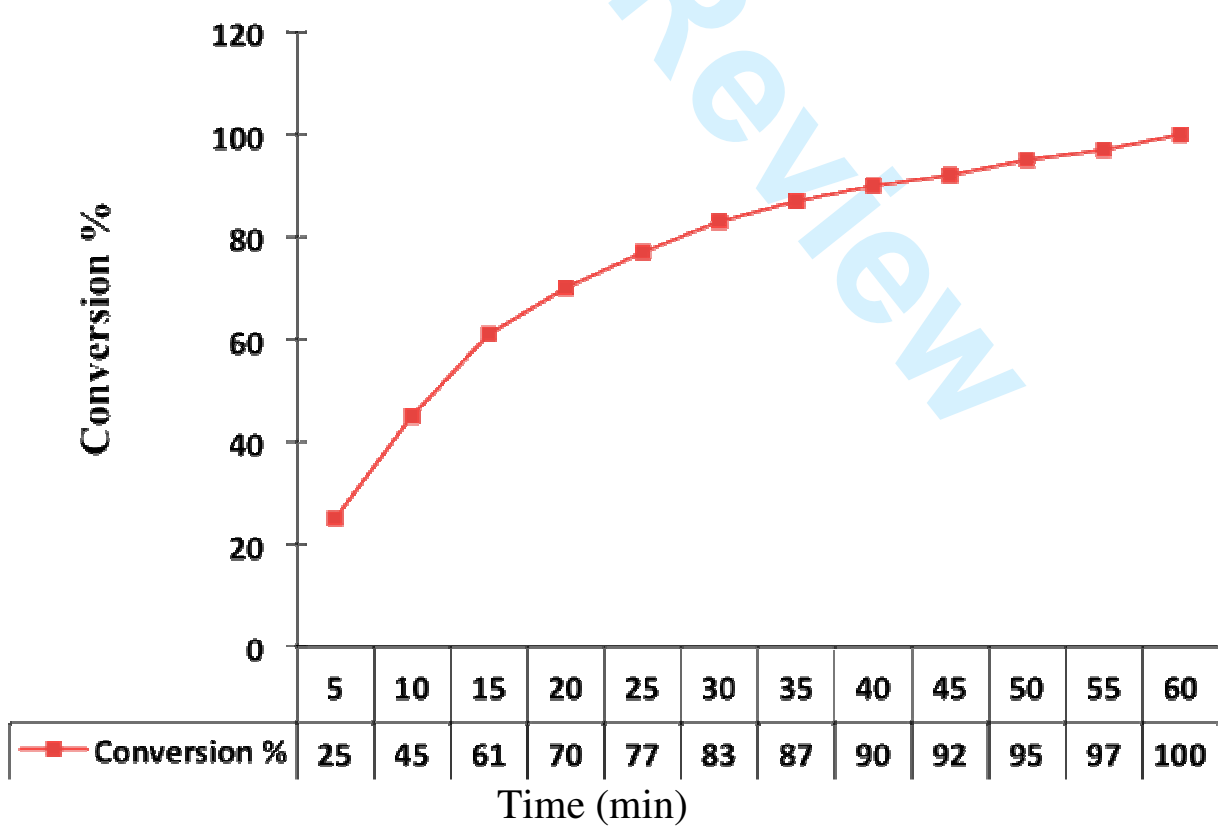

Figure 4: Plot of conversion versus time for $p$-methylacetophenone $(1 \mathrm{mmol})$ catalysed by $9 \mathbf{c}(0.3 \mathrm{~mol} \%)$ 


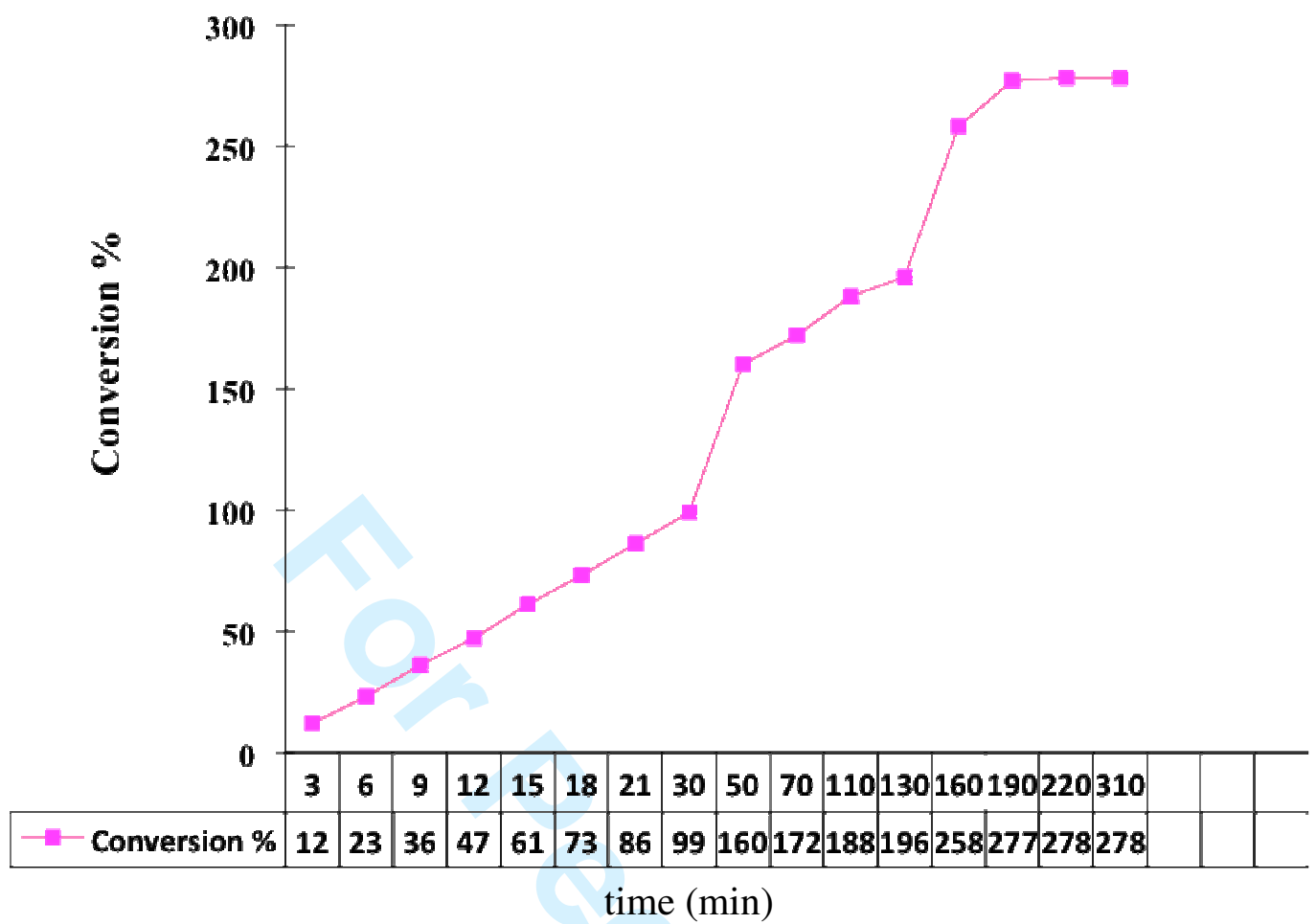

Figure 5. Lifetime studies for catalyst 9 c $(0.3 \mathrm{~mol} \%)$ with $p$-bromocetophenone as substrate 